\title{
KUWABARA-KONO NUMERICAL DISSIPATION: A NEW METHOD TO SIMULATE GRANULAR MATTER
}

\author{
GUILLAUME JAMES $^{1,2}$, KIRILL VOROTNIKOV $^{1,2}$, BERNARD BROGLIATO $^{1,2}$
}

\begin{abstract}
We introduce a new method for the simulation of multiple impacts in granular media described by the Kuwabara-Kono (KK) model, a nonsmooth (not Lipschitz continuous) extension of Hertz contact that accounts for viscoelastic damping. We use the technique of modified equations to construct time-discretizations of the nondissipative Hertz law matching numerical dissipation with KK dissipation at different consistency orders. This allows us to simulate dissipative impacts with good accuracy without including the nonsmooth viscoelastic component in the contact force. This tailored numerical dissipation is developed in a general framework, for Newtonian dynamical systems subject to dissipative forces proportional to the time-derivative of conservative forces. Numerical tests are performed for the simulation of impacts in Newton's cradle and on alignments of alternating large and small balls. Resulting wave phenomena (oscillator synchronisation, propagation of dissipative solitary waves, oscillatory tails) are accurately captured by implicit schemes with tailored numerical dissipation, even for relatively large time steps.
\end{abstract}

\section{INTRODUCTION}

Analyzing the response of granular media to impacts and vibrations is a fundamental issue in many contexts, e.g. to understand crater formation [1], model the response of ballast to moving loads [2], or to design shock absorbers $[3,4]$ and more general granular metamaterials for impact redirection, acoustic lensing or filtering $[5,6,7]$. In particular, one-dimensional granular media (such as the granular chain depicted in figure 1) have been extensively studied. Despite their apparent simplicity, it is a difficult problem to understand the dispersion and dissipation of energy in these systems [8] and to analyze related wave effects [9], in particular the formation of solitary waves and breathers $[10,11,12,13,14$, $15]$.

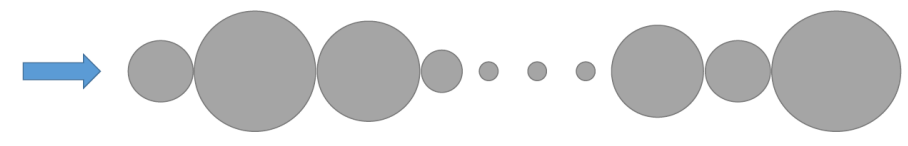

FIGURE 1. Schematic representation of a granular chain, with an impulsive force applied to the first element.

Multiple impacts in multibody mechanical systems such as chains of balls can be studied using different types of models. Algebraic models that relate post- and pre-impact velocities under the assumption of rigid body collisions and instantaneous impacts are widely used, due to their high numerical tractability (see $[8,16,17]$ for reviews). However, neglecting compliance and wave effects in multiple impacts often leads to strong discrepancies with experimental results and more realistic models $[8,18]$.

Date: September 21, 2018.

Key words and phrases. Granular chains, Newton's cradle, viscoelastic impact models, implicit Runge-Kutta methods, numerical dissipation, modified equation, solitary waves. 
Another classical approach is based on second-order dynamics and compliant contact models. According to Hertz's theory, the repulsive force $f$ between two initially tangent spherical beads (or smooth non-conforming surfaces) compressed with a small relative displacement $\delta$ is $f(\delta)=k \delta_{+}^{3 / 2}$ at leading order in $\delta$, where $\delta_{+}=\max (\delta, 0)$ (no force is present when beads are not in contact) and $k$ depends on the radii of curvature and elastic coefficients of the balls [19]. Moreover, energy loss arising from viscoelasticity (and from the excitation of elastic vibrations to some extent) is generally taken into account through a contact law of the type

$$
f(\delta)=k\left(\delta_{+}^{3 / 2}+\gamma \frac{d}{d t} \delta_{+}^{\beta}\right)
$$

depending on parameters $\gamma>0$ and $\beta \geq 1$ (see e.g. [17, 20, 21] for reviews). Classical choices correspond to $\beta=1$ (Hertzian spring with linear dashpot [22]), $\beta=5 / 2$ (Simon-Hunt-Crossley model [23, 24]), and $\beta=3 / 2$ which leads to the Kuwabara-Kono (KK) model [25] (see also [26] for a ball impacting a plate). This approach applies to small impact velocities and does not account for energy loss due to plastic deformations (see [27, 28, 8, 29, 30] for impact laws incorporating plasticity and their application to granular chains). The above compliant dissipative models agree reasonably well with several experiments on binary collisions [21, 25, 31, 32] and impacts on granular chains $[33,34]$, and an optimal choice of model seems to depend actually on the experimental configuration [35]. However, the KK model has been put on a more rigorous footing compared to the other (more phenomenological) impact laws because it can be derived from continuum mechanics (see $[36,37]$ and references therein). In particular, the associated damping constant $\gamma$ is determined by the elastic and viscous coefficients of the materials and can be inferred from experiments.

The numerical simulation of compliant models can be delicate for large systems or if the dynamical equations are numerically stiff. This situation arises for spatially inhomogeneous systems when masses or stiffness constants vary strongly, or when strongly compressed regions coexist with loosely compressed elements (or beads in free flight), leading usually to different time scales. Another difficulty arises from the limited smoothness of the Hertz contact force, which is $C^{1}$ but not $C^{2}$ at the point of contact breaking (i.e., $\delta=0$ ). The situation can be even worse for the viscoelastic impact laws, since the contact force is discontinuous at $\delta=0$ for $\beta=1$, and is not Lipschitz continuous for $\beta \in(1,2)$ (in particular for the KK model). This lack of smoothness tends to limit the order of convergence of timeintegration methods, and some authors recommend to use at least 1000 discretization points during a collision [17].

In this work, we introduce a new method for the numerical simulation of granular chains which allows to approximate the KK model without explicitly including the nonsmooth viscoelastic term in the contact force. Our approach, thereafter denoted as tailored numerical dissipation, applies more generally to Newtonian dynamical systems for which dissipative forces are given by the time-derivative of conservative forces with some prefactor $\gamma$ (as in the case $\beta=3 / 2$ of (1)). Moreover, the method can be declined within a variety of numerical schemes, in particular implicit ones allowing for relatively large step sizes. Our approach is based on the technique of modified (or equivalent) equations (see e.g. $[38,39]$ and references therein), in which effects induced by time-discretization (such as numerical dissipation) are analyzed by considering suitable perturbations of the initial differential equation. Considering a conservative Newtonian system approximated by a general two-stage Runge-Kutta (RK) method [40] (including the $\theta$ method), we derive a dissipation condition under which the modified equation coincides with the dissipative perturbation at leading order in the time step $h$. This condition relates $h$ and the scheme parameters to a small damping constant $\gamma$ in the dissipative model. In particular, starting from a nondissipative granular chain with Hertzian interactions, one can tune scheme parameters in order to correctly capture KK dissipation using numerical dissipation only, i.e., neglecling the viscoelastic component in the contact force. Furthermore, we construct a time-discretization of the conservative system which is consistent at order 3 with the dissipative model and takes the form 
of an implicit Runge-Kutta (IRK) method with tailored numerical dissipation. This scheme corresponds to a perturbed Gauss-Legendre (GL) scheme [40] and is far more precise than the second order schemes that satisfy solely the above dissipation condition, a result that we observe even for relatively large step sizes.

This approach can be generalized in order to combine different types of dissipation. As an illustration, we consider a Newton's cradle $[34,15]$ in which the granular chain of figure 1 is modified by adding a local attachment to each bead, which induces a local (quadratic) potential. Using a twoparameter additive $\theta$ method (a particular additive RK method [41]), we show that numerical dissipation can match arbitrary values of local (linear) and contact (KK) dampings. In particular, one can set the scheme parameters to obtain a vanishing local damping. In that case, in the time-discretization of the conservative Newton's cradle, numerical dissipation is able to reproduce an asymptotic synchronisation of the oscillators that occurs with the KK model [34].

Note that there exists another approach to quantify numerical dissipation, which is based on dissipation equalities for suitable discrete energies, see e.g. [42] and references therein. However, our approach has the advantage of estimating the consistency order and allows us to obtain error bounds. As an illustration, for a spatially homogeneous granular chain with Hertz contact law, we prove the convergence of the above $\theta$ method towards the KK model with $\gamma=O(h)$. We derive a $O\left(h^{3 / 2}\right)$ estimate for the global error, which lies above the $O\left(h^{2}\right)$ bound expected from a standard consistency analysis, a difference which is due to the lack of regularity of the KK model.

The above approach based on tailored numerical dissipation is compared to direct time-discretizations of the dissipative models. We simulate an impact on a granular chain, where the KK model is approximated either using a Crank-Nicolson $(\mathrm{CN})$ scheme or a GL scheme. When the classical positionvelocity formulation is used, we numerically observe an order of convergence limited to $3 / 2$ for both schemes (whereas the usual order is 2 for the CN scheme and 4 for the GL scheme). This order reduction is due to the fact that the $\mathrm{KK}$ model is not Lipschitz continuous. In order to attenuate this phenomenon, one has to introduce a change of variables leading to more regular dynamical equations, for which the $\mathrm{CN}$ scheme recovers the order 2 and the GL scheme has order $5 / 2$. These schemes are compared to the $\theta$ and IRK schemes with tailored numerical dissipation in the case of an impact on a dissipative dimer chain (i.e., aligned beads with alternatively large and small radii). This problem constitutes an interesting test because the impact generates different types of solitary-like waves (with a small or large amount of dispersion) depending on the mass ratio between beads $[12,8,18]$. These waves are qualitatively well described both by tailored numerical dissipation and direct discretization, even for relatively large time steps for the IRK and GL schemes. When the time step is small, we obtain the most precise results with direct discretization of the KK model written in its regularized form. However, for rather large time steps and $\gamma$ not too large, tailored numerical dissipation is competitive with the direct discretizations, and the IRK scheme is even more precise than the GL scheme in some physical range of damping constants $\gamma$.

The structure of the paper is as follows : in section 2, we recall basic properties of the KK model, establish the well-posedness of the dynamical equations for the contact laws (1) including the nonsmooth cases, and study numerically the orders of the CN and GL schemes for different formulations of the KK model. In section 3, we present the approach of tailored numerical dissipation in a general framework. Section 3.1 recalls the main features of modified equations for one-step methods applied to autonomous nonlinear ODE. We introduce the additive $\theta$ method (section 3.2) and IRK scheme (sections 3.3 and 3.4) with tailored numerical dissipation, and study numerically their orders of convergence for the KK model (section 3.5). Section 4 concerns the simulation of impacts in dimer chains and the comparison of the different numerical schemes. Section 5 summarizes the above results and draws some perspectives opened by this study. Finally, different technical results are presented in the appendix, where we establish the $A$-stability [40] of the IRK scheme with tailored dissipation (section A.1) and prove the convergence of the $\theta$ method with KK numerical dissipation (section A.2). 


\section{DYNAMiCAL EQUATIONS FOR GRANULAR CHAINS}

We focus on dissipative granular chains without precompression. The chains are made of $N$ spherical elements (with masses $m_{1}, \ldots, m_{N}$ ) interacting through the contact law (1), with stiffness and damping parameters $k_{n}, \gamma_{n}$ for the contact between elements $n-1, n$. The dynamical equations are

$$
\begin{aligned}
m_{n} \ddot{x}_{n}= & k_{n}\left(x_{n-1}-x_{n}\right)_{+}^{3 / 2}+\beta \gamma_{n} k_{n}\left(x_{n-1}-x_{n}\right)_{+}^{\beta-1}\left(\dot{x}_{n-1}-\dot{x}_{n}\right) \\
& -k_{n+1}\left(x_{n}-x_{n+1}\right)_{+}^{3 / 2}-\beta \gamma_{n+1} k_{n+1}\left(x_{n}-x_{n+1}\right)_{+}^{\beta-1}\left(\dot{x}_{n}-\dot{x}_{n+1}\right), \quad 1 \leq n \leq N,
\end{aligned}
$$

where $x_{n}$ is the displacement of the center of the $n^{\text {th }}$ element from its equilibrium position (corresponding to an uncompressed chain of touching beads) and overdots denote time derivatives. The chain ends are free, i.e., we set $k_{1}=k_{N+1}=0$ in (2). In most of the numerical simulations of (2), we shall consider the initial condition

$$
x_{n}(0)=0 ; \quad \dot{x}_{1}(0)=V_{\text {imp }}, \quad \dot{x}_{n}(0)=0 \forall n \geq 2,
$$

corresponding to the first bead impacting the others (initially at rest) at velocity $V_{\text {imp }}>0$.

In the sequel, we start by recalling basic properties of the KK model (case $\beta=3 / 2$ of (2)), establish the well-posedness of the evolution problem (2) in the general case, and explore the behavior of different numerical time-integration schemes applied to the KK model.

2.1. Kuwabara-Kono model. In this work, the analysis of impacts will be performed for $\beta=3 / 2$ in (2) and a constant damping parameter $\gamma$. This is the case when all beads are made with the same material or for dimer chains alternating two types of beads. In that case system (2) becomes

$$
\begin{aligned}
m_{n} \ddot{x}_{n}= & k_{n}\left(x_{n-1}-x_{n}\right)_{+}^{3 / 2}+\frac{3}{2} \gamma k_{n}\left(x_{n-1}-x_{n}\right)_{+}^{1 / 2}\left(\dot{x}_{n-1}-\dot{x}_{n}\right) \\
& -k_{n+1}\left(x_{n}-x_{n+1}\right)_{+}^{3 / 2}-\frac{3}{2} \gamma k_{n+1}\left(x_{n}-x_{n+1}\right)_{+}^{1 / 2}\left(\dot{x}_{n}-\dot{x}_{n+1}\right), \quad 1 \leq n \leq N .
\end{aligned}
$$

It is interesting to consider the particular case of two beads $(N=2)$ and study the restitution coefficient $e$ defined as the ratio of (the moduli of) post- and pre-impact relative velocities. Considering the relative displacement $\delta=x_{1}-x_{2}$, equation (4) leads to

$$
m \ddot{\delta}=-k \delta_{+}^{3 / 2}-\frac{3}{2} \gamma k \delta_{+}^{1 / 2} \dot{\delta},
$$

where we denote by $m=\frac{m_{1} m_{2}}{m_{1}+m_{2}}$ the reduced mass and $k=k_{2}$. The initial condition (3) yields $\delta(0)=0$ and $\dot{\delta}(0)=V_{\mathrm{imp}}$. Moreover, one can rewrite (5) in dimensionless form by setting

$$
T=\left(\frac{m}{k}\right)^{2 / 5} V_{\text {imp }}^{-1 / 5}
$$

$t=\tau T$ and $\delta(t)=V_{\mathrm{imp}} T \zeta(\tau)$. This leads to the renormalized equation

$$
\frac{d^{2} \zeta}{d \tau^{2}}=-\zeta_{+}^{3 / 2}-\frac{3}{2} \tilde{\gamma} \zeta_{+}^{1 / 2} \frac{d \zeta}{d \tau}
$$

with dimensionless damping parameter

$$
\tilde{\gamma}=\gamma V_{\mathrm{imp}}^{1 / 5}\left(\frac{k}{m}\right)^{2 / 5}
$$

and the initial condition $\zeta(0)=0, \frac{d \zeta}{d \tau}(0)=1$. The restitution coefficient can then be expressed as

$$
e=\left|\frac{d \zeta}{d \tau}\left(\tau_{c}\right)\right|
$$

where $\tau_{c}$ is the collision duration. When $\tilde{\gamma} \approx 0$ (or equivalently $e \approx 1$ ) one has (see e.g. [43])

$$
\tilde{\gamma} \approx 0.578(1-e) \text {. }
$$


For example, restitution coefficients obtained experimentally with different types of beads [25] for $V_{\mathrm{imp}}=0.5 \mathrm{~m} \mathrm{~s}^{-1}$ suggest $\tilde{\gamma} \approx 0.01$ for glass spheres (radius $R=1.96 \mathrm{~cm}$ ), $\tilde{\gamma} \approx 0.06$ for steel spheres $(R=1.65 \mathrm{~cm})$, and $\tilde{\gamma} \approx 0.17$ for cork spheres with a lead core $\left(R=1.66 \mathrm{~cm}, m_{n}=31.7 \mathrm{~g}\right)$.

In the forthcoming sections, we shall work with equation (4) rescaled in a similar form obtained by setting $m=m_{1}$ and $k=k_{2}$ in (6). Moreover, we will consider relatively small values of the rescaled damping constant (8), consistently with the above estimates.

2.2. Well-posedness and time-discretizations of the dissipative contact problem. The well-posedness of the initial value problem for equation (2) is not immediate for $\beta \in[1,2)$, since the map defining the right-hand side of (2) is discontinuous accross the hyperplanes $x_{n-1}=x_{n}$ for $\beta=1$, and not Lipschitz continuous for $\beta \in(1,2)$ (in particular for the KK model).

This difficulty can be circumvented by an appropriate choice of dynamical variables. Defining a generalized velocity $w_{n}$ through

$$
\dot{x}_{n}=w_{n}+\frac{1}{m_{n}}\left(\gamma_{n} k_{n}\left(x_{n-1}-x_{n}\right)_{+}^{\beta}-\gamma_{n+1} k_{n+1}\left(x_{n}-x_{n+1}\right)_{+}^{\beta}\right),
$$

the dynamical equation (2) becomes

$$
\dot{w}_{n}=\frac{1}{m_{n}}\left(k_{n}\left(x_{n-1}-x_{n}\right)_{+}^{3 / 2}-k_{n+1}\left(x_{n}-x_{n+1}\right)_{+}^{3 / 2}\right) .
$$

Now let us denote $x=\left(x_{1}, \ldots, x_{N}\right), w=\left(w_{1}, \ldots, w_{N}\right)$ and $Y=(x, w)$. The map $(x, \dot{x}) \mapsto Y$ defines an homeomorphism in $\mathbb{R}^{2 N}$ when $\beta>0$. Moreover, for all $\beta \geq 1$, the right-hand side of (10)-(11) defines a locally Lipschitz continuous map $Y \mapsto F_{\mathrm{s}}(Y)\left(F_{\mathrm{s}}\right.$ is even $C^{1}$ for $\left.\beta>1\right)$ and thus the corresponding initial value problem is well posed according to the Cauchy-Lipschitz theorem. Moreover, since the energy

$$
H(t)=\frac{1}{2} \sum_{n=1}^{N} m_{n} \dot{x}_{n}^{2}(t)+\frac{2}{5} \sum_{n=2}^{N} k_{n}\left(x_{n-1}(t)-x_{n}(t)\right)_{+}^{5 / 2}
$$

is nonincreasing along any solution, it follows that $\dot{x}_{n}(t)$ is bounded and $\left|x_{n}(t)\right|$ grows at most linearly for all $n=1, \ldots, N$ and all positive times, hence all solutions are globally defined for $t \geq 0$.

The new formulation (10)-(11) is also very useful for the purpose of numerical integration. Indeed, for several numerical schemes, the improved regularity of the right-hand side brings better convergence properties compared to the differential equation based on the usual dynamical variables $\left(x_{n}, \dot{x}_{n}\right)$, whose right-hand side is not Lipschitz continuous for $\beta \in[1,2)$.

In order to illustrate this point, let us consider a chain of three spherical beads (of the same material) having different radii and use the KK model (4) rewritten in dimensionless form. Masses are set to $m_{n}=R_{n}^{3}$ with $R_{1}=1, R_{2}=4 / 5, R_{3}=9 / 10$ ( $R_{n}$ corresponds to a rescaled bead radius), and the rescaled Hertzian stiffness [19] at the different contacts is $k_{2}=1, k_{3}=\sqrt{81 / 85}$. We fix $\gamma=0.1$.

Reference solutions $X=(x, \dot{x})$ are computed using the standard ODE solver of the software package Scilab, with relative and absolute error tolerances set to $10^{-13}$ and $10^{-15}$, respectively. In that case, the numerical time-integration of the KK model is performed using formulation (10)-(11) for $Y(t)$ (with $\beta=3 / 2$ ), and velocity components of $X(t)$ are recovered from the dynamical variable $Y(t)$ through identity (10).

For several initial conditions $X(0)=X_{0}$ and different time steps $h$, we compare the reference solution $X(k h)$ to numerical approximations $X_{k}$ obtained using four different methods, which correspond to IRK schemes with different orders. The first two schemes are applied to (4) rewritten in the form $\dot{X}=F(X)$, i.e., using the usual dynamical variables corresponding to bead positions and velocities. We consider the Crank-Nicolson (CN) scheme (or trapezoidal rule) [40, 44] defined by

$$
\frac{X_{k+1}-X_{k}}{h}=\frac{1}{2}\left(F\left(X_{k+1}\right)+F\left(X_{k}\right)\right) \text {. }
$$


This defines a second-order method when the dynamical equations are sufficiently smooth (i.e., the right-hand side is $C^{2}$ ), but such estimates do not apply in our case since $F$ is not Lipschitz continuous. In addition, we consider the Gauss-Legendre (GL) method $[45,46]$ defined by

$$
\frac{X_{k+1}-X_{k}}{h}=\frac{k_{1}+k_{2}}{2},
$$

where $k_{1}, k_{2}$ are determined from the implicit equations

$$
k_{i}=F\left(X_{k}+h\left(a_{i 1} k_{1}+a_{i 2} k_{1}\right)\right), \quad i=1,2,
$$

with

$$
a_{11}=a_{22}=\frac{1}{4}, \quad a_{12}=\frac{1}{4}-\frac{\sqrt{3}}{6}, \quad a_{21}=\frac{1}{4}+\frac{\sqrt{3}}{6} .
$$

This defines a fourth-order method in the case of sufficiently smooth systems (i.e., if the right-hand side is $C^{4}$ ), but this result does not apply to the vector field $F$. The two remaining schemes are applied to the equation $\dot{Y}=F_{\mathrm{S}}(Y)$ corresponding to system (10)-(11). Approximations $Y_{k}$ to $Y(k h)$ are obtained with the $\mathrm{CN}$ scheme

$$
\frac{Y_{k+1}-Y_{k}}{h}=\frac{1}{2}\left(F_{\mathrm{s}}\left(Y_{k+1}\right)+F_{\mathrm{S}}\left(Y_{k}\right)\right)
$$

and the GL method

$$
\begin{gathered}
\frac{Y_{k+1}-Y_{k}}{h}=\frac{k_{1}+k_{2}}{2}, \\
k_{i}=F_{\mathrm{s}}\left(Y_{k}+h\left(a_{i 1} k_{1}+a_{i 2} k_{1}\right)\right), \quad i=1,2,
\end{gathered}
$$

with coefficients $a_{i j}$ given in (15). Using the resulting approximations of $x(k h)$ and $w(k h)$ in conjunction with identity (10) for the velocity components, one obtains approximations $X_{k}$ to $X(k h)$.

At each iteration, the implicit equations that define each scheme are solved using the Scilab fsolve function (based on a Powell hybrid method) with relative error tolerance set to $10^{-13}$. The time evolution of bead positions and velocities is approximated in an interval $\left[0, T_{\max }\right]$, and for each scheme we compute the global error $E=\max _{k \in\left[0, T_{\max }\right]}\left\|X(k h)-X_{k}\right\|_{\infty}$.

We first consider the initial condition (3) with impact velocity $V_{\text {imp }}=1$, and we set the maximal time of integration to $T_{\max }=5$ (slightly above the duration of the multiple impact). The global errors resulting from the four different schemes are given in figure 2. Interestingly, the CN scheme (16) based on the more regular dynamical equations keeps its optimal order 2 (this is not automatic, since $F_{\mathrm{s}}$ is $C^{1,1 / 2}$ and not $C^{2}$ ). The situation is different for the CN scheme (12) based on the less regular ODE. In that case, while the order seems close to 2 for relatively large $h$, there is a crossover around $h=10^{-2}$, and below this step size the order drops to $3 / 2$. Order reduction is also observed for the GL scheme. The scheme (13)-(14) based on the less regular ODE has only order $3 / 2$, which limits its interest due to its higher computational cost (the dimension of the nonlinear algebraic system solved at each iteration is twice the dimension of the CN system). The same phenomenon occurs with the scheme (17)-(18) applied to the smoother dynamical equations, but order reduction is less important since the scheme has order $5 / 2$. We also note a saturation of the global error around $10^{-12}$ when $h$ reaches $10^{-4}$, followed by an increase of the error to $10^{-11}$ when $h$ is further decreased to $10^{-5}$. Such behavior is typically linked with the error tolerances of the Scilab ode and fsolve functions and the accumulation of errors in double-precision arithmetics, but it is certainly enhanced by the limited smoothness of the $C^{1,1 / 2}$ map $F_{\mathrm{s}}$. Interestingly, we also observe that this GL scheme remains quite precise even for rather large values of $h$, as illustrated by the bottom panels of figure 2. Finally, in the case of the GL schemes, we also note that the global error does not decay monotonically when $h$ goes to 0 and exhibits small oscillations, a phenomenon which may be linked with the limited smoothness of the dynamical equations.

The above order reduction phenomena are due to the limited smoothness of the maps $F$ and $F_{\mathrm{s}}$, which shows up only in the case of contact creation or breaking. To illustrate this point, let us consider 

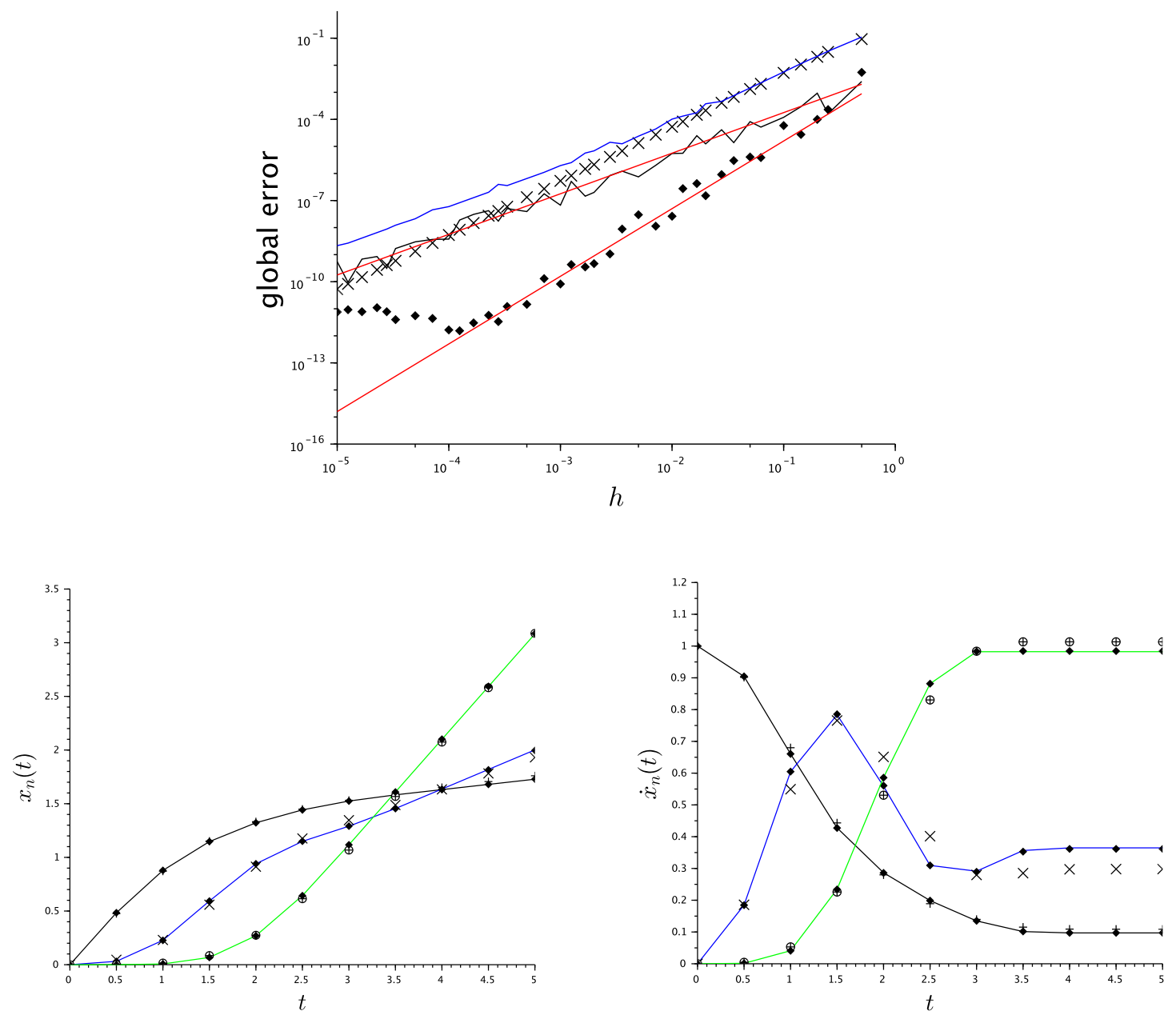

FIGURE 2. Top : global error $E$ computed versus step size $h$ for four different methods : CN schemes (12) (blue line) and (16) (crosses), GL schemes (13)-(14) (black line) and (17)-(18) (diamonds). The errors are plotted in logarithmic scale. The red lines are plotted to guide the eye and correspond to $E=a h^{3 / 2}$ (top line) and $E=b h^{5 / 2}$ (bottom line) with appropriate fitting parameters $a, b$. We consider the initial condition (3) with $V_{\mathrm{imp}}=1$, and solutions are computed for $t \in[0,5]$. Bottom : displacements (left panel) and velocities (right panel) of beads with index $n=1$ (black line), $n=2$ (blue), $n=3$ (green), and their approximations by the $\mathrm{CN}$ scheme (16) (crosses) and GL scheme (17)-(18) (black diamonds) for $h=0.5$.

the new initial condition and maximal time of integration

$$
x(0)=(0.9,0.2,0), \quad \dot{x}(0)=(0.7,0.6,0.02), \quad T_{\max }=1.5,
$$

so that no contact breaking occurs on $\left[0, T_{\max }\right]$ (see the right panel of figure 3 ). The global errors resulting from the four different schemes are given in the left panel of figure 3 . One can see that all schemes keep their optimal orders ( 2 for the CN schemes, and 4 for the GL methods). This not surprising since $F$ and $F_{\mathrm{S}}$ define analytic maps in the corresponding regions of the phase space where all beads remain in contact. One can notice that the global error saturates around $10^{-13}$ for the GL schemes 
with time steps below $10^{-3}$, but this saturation effect is less important compared to the situation of figure 2. Moreover, the results are extremely close if dynamical equations are discretized at the level of $X(t)$ or $Y(t)$.
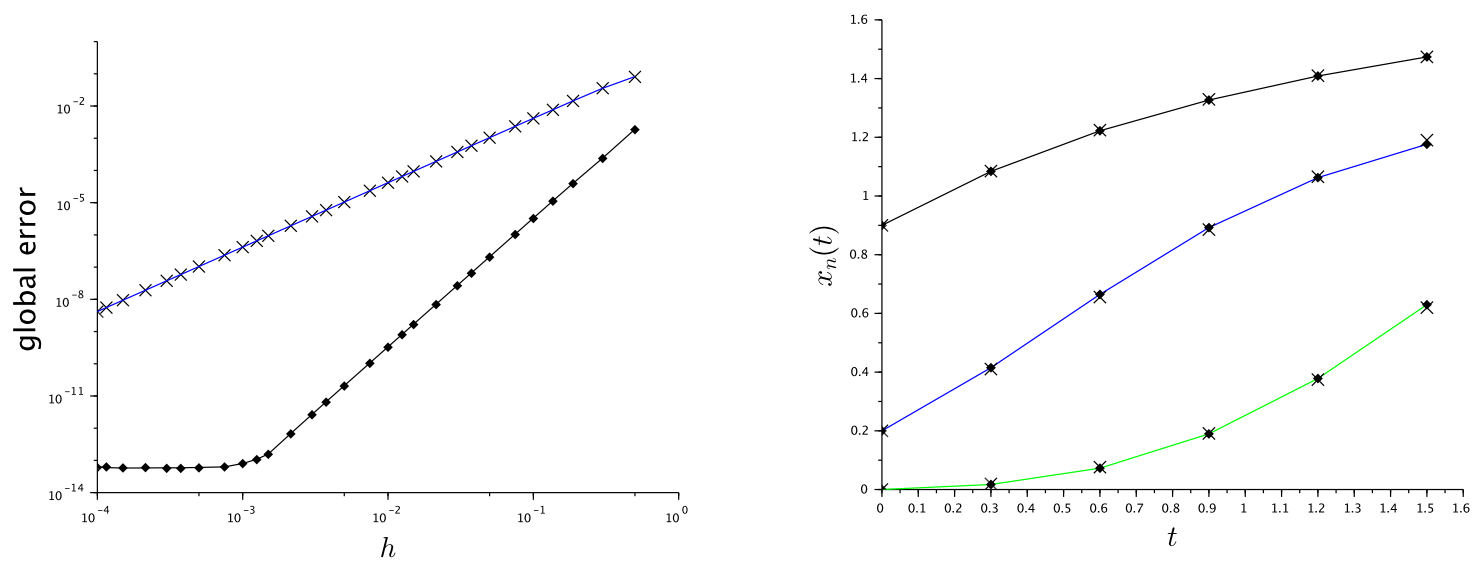

FIGURE 3. The left panel provides the global error computed versus step size $h$ for four different methods : CN schemes (12) (blue line) and (16) (crosses), GL schemes (13)-(14) (black line) and (17)-(18) (diamonds). The errors are plotted in logarithmic scale. The initial condition and maximal time of integration are given in (19). The right panel displays the displacements of beads with index $n=1$ (black line), $n=2$ (blue), $n=3$ (green), and their approximations by the $\mathrm{CN}$ scheme (16) (crosses) and GL scheme (17)-(18) (diamonds) for $h=0.3$.

In conclusion, in situations where contact creation or breaking occurs, it is preferable to discretize the dynamical equations rewritten in the form (10)-(11) rather that using the usual dynamical variables $\left(x_{n}, \dot{x}_{n}\right)$.

\section{TAILORED NUMERICAL DISSIPATION AND ITS APPLICATION TO THE KUWABARA-KONO MODEL}

Given a smooth ODE discretized with a one-step method of order $p$, there exists a suitable small perturbation of the equation, thereafter denoted as modified equation, which is consistent with the numerical scheme at an order $m>p$ ( $m$ can be chosen arbitrarily large if the ODE is infinitely smooth). In this section, we show that the KK model with $\gamma=O(h)$ arises as a modified equation for different dissipative numerical schemes used to approximate impacts in nondissipative granular chains. This observation leads to a new class of time-integration methods with tailored numerical dissipation. The schemes correspond to an additive $\theta$ method $(p=1, m=2)$ and two-stage IRK methods $(p=1$, $m=2$ or 3 depending on the scheme).

We start by recalling general properties of modified equations in section 3.1. In section 3.2, the dynamical equations of Newton's cradle are discretized with the additive $\theta$ method. The class of twostage IRK methods is studied in sections 3.3 and 3.4. The orders of the methods are numerically tested in section 3.5 for a granular chain.

3.1. General properties of modified equations. We consider an autonomous differential equation in $\mathbb{R}^{d}$

$$
\frac{d y}{d t}=f(y)
$$


where the right-hand side $f$ is assumed sufficiently smooth. Given a smooth map $g: \mathbb{R}^{d} \rightarrow \mathbb{R}^{d}$, we denote by $L_{f} g(y)=D g(y) f(y)$ the Lie derivative of $g$ along $f$ ( $D g$ denotes the Jacobian matrix of $g$ ). More generally, we denote by $L_{f}^{k} g$ the $k$ th Lie derivative of $g$ along $f$, which is defined by induction through $L_{f}^{k} g=L_{f} L_{f}^{k-1} g$.

We discretize (20) with a general one-step method

$$
\frac{Y_{k+1}-Y_{k}}{h}=\phi\left(Y_{k}, h\right)
$$

where $h$ denotes the time step, $Y_{k}$ an approximation of $y(k h)$, and the application $\phi: \mathbb{R}^{d} \times \mathbb{R} \rightarrow \mathbb{R}^{d}$ is assumed sufficiently smooth. In this paper we shall resort to implicit schemes, hence $\phi$ will be defined implicitly through a system of nonlinear equations solved at each time step.

We assume $\phi(Y, 0)=f(Y)$, so that the scheme (21) is consistent with (20). Denoting by $p \geq 1$ the order of the scheme, one has for all solution $y$ of (20) and $h \rightarrow 0$ :

$$
R_{y, h}(t)=O\left(h^{p}\right),
$$

where $R_{y, h}$ is the consistency error defined by :

$$
R_{y, h}(t)=\frac{y(t+h)-y(t)}{h}-\phi(y(t), h) .
$$

Property (22) is equivalent to the following condition (see e.g. [47]) :

$$
\partial_{h}^{k} \phi(y, 0)=\frac{1}{k+1} L_{f}^{k} f(y), \quad 0 \leq k \leq p-1 .
$$

Now let us consider a smooth map $\mathcal{F}: \mathbb{R}^{d} \times \mathbb{R} \rightarrow \mathbb{R}^{d}$ satisfying $\mathcal{F}(y, 0)=f(y)$ and the perturbed system

$$
\frac{d Y}{d t}=\mathcal{F}(Y, h)
$$

Given an integer $m>p$, we search for $\mathcal{F}$ such that the scheme (21) is consistent at the higher order $m$ with (25). This means that for all solution $Y$ of (25) one has

$$
R_{Y, h}(t)=O\left(h^{m}\right)
$$

when $h \rightarrow 0$. Equation (25) will be denoted as a modified equation at the mth order relative to the scheme, or simply modified equation.

Typically, perturbative terms in (25) allow to capture possible effects of time-discretization such as numerical dissipation or instabilities. A thorough description of the theory of modified equations can be found in chapter IX of [39] to which we refer for details. We summarize below some basic results that will be useful for our purpose.

Modified equations are clearly non-unique, since property (26) remains true for any smooth $O\left(h^{m}\right)$ perturbation of the right-hand side in (25). However, all modified equations at the $m$ th order admit the same Taylor expansion in $h$ at order $m-1$. Its coefficients are determined by induction from condition (26), where an expansion of $R_{Y, h}$ with respect to $h$ can be obtained using the expansions of $\phi,(25)$ and its time derivatives. This yields the Taylor expansion of $\mathcal{F}$ :

$$
\mathcal{F}(Y, h)=f(Y)+\sum_{j=p}^{m-1} h^{j} F_{j}(Y)+O\left(h^{m}\right),
$$

where the leading order correction term is given by

$$
F_{p}=\frac{1}{p !}\left(\partial_{h}^{p} \phi-\frac{1}{p+1} L_{f}^{p} f\right) .
$$


A recurrence formula for the coefficients $F_{j}$ is given in [39] (Lemma 7.3 p.362). Introducing $\phi_{k}=$ $\frac{1}{k !} \partial_{h}^{k} \phi$, one has in particular :

$$
\begin{gathered}
F_{1}=\phi_{1}-\frac{1}{2} L_{f} f, \\
F_{2}=\phi_{2}-\frac{1}{6} L_{f}^{2} f-\frac{1}{2}\left(L_{f} F_{1}+L_{F_{1}} f\right) .
\end{gathered}
$$

The explicit computation of $F_{j}$ becomes cumbersome for large $j$ or when the coefficients $\phi_{k}$ are heavy to compute, but the procedure can be automated using a computer algebra software [48].

It is interesting to note that the coefficient (28) appears at leading order in the consistency error (22), where $R_{y, h}=-h^{p} F_{p}(y)+O\left(h^{p+1}\right)$ ([39], Theorem 1.2 p.340).

3.2. An additive $\theta$ method with Kuwabara-Kono numerical dissipation. We consider a Newton's cradle, which corresponds to a granular chain of the same type as in figure 1, but including local attachments consisting of strings [34] or plates [15]. The dynamical equations correspond to a generalization of system (4) including local restoring forces, where the attachment of the $n$th bead is represented by a spring with linear stiffness $K_{n}$, namely :

$$
m_{n} \ddot{x}_{n}=-K_{n} x_{n}+k_{n} \delta_{(n-1)+}^{3 / 2}-k_{n+1} \delta_{n+}^{3 / 2}, \quad 1 \leq n \leq N,
$$

with $\delta_{n}=x_{n}-x_{n+1}$ and $k_{1}=k_{N+1}=0$. In model (31), collisions are assumed nondissipative (KK dissipation will be taken into account at a later stage). Setting $x=\left(x_{1}, \ldots, x_{N}\right), v=\left(\dot{x}_{1}, \ldots, \dot{x}_{N}\right)$ and $y=(x, v)$, system (31) can be reformulated as

$$
\dot{y}=f(y)=\left(\begin{array}{c}
v \\
g(x)
\end{array}\right),
$$

where the components of $g(x) \in \mathbb{R}^{N}$ are given by

$$
g_{n}(x)=\frac{1}{m_{n}}\left(-K_{n} x_{n}+k_{n} \delta_{(n-1)+}^{3 / 2}-k_{n+1} \delta_{n+}^{3 / 2}\right) .
$$

In order to approximate solutions of (32), we introduce the implicit one-step method

$$
\begin{aligned}
\frac{X_{n}^{k+1}-X_{n}^{k}}{h}= & \theta V_{n}^{k+1}+(1-\theta) V_{n}^{k}, \\
\frac{V_{n}^{k+1}-V_{n}^{k}}{h}= & \frac{1}{m_{n}}\left[-(1-\mu) K_{n} X_{n}^{k+1}+\theta\left(k_{n}\left(\Delta_{n-1}^{k+1}\right)_{+}^{3 / 2}-k_{n+1}\left(\Delta_{n}^{k+1}\right)_{+}^{3 / 2}\right)\right] \\
& +\frac{1}{m_{n}}\left[-\mu K_{n} X_{n}^{k}+(1-\theta)\left(k_{n}\left(\Delta_{n-1}^{k}\right)_{+}^{3 / 2}-k_{n+1}\left(\Delta_{n}^{k}\right)_{+}^{3 / 2}\right)\right],
\end{aligned}
$$

where $\Delta_{n}^{k}=X_{n}^{k}-X_{n+1}^{k}, h$ denotes the time step and $Y_{k}=\left(X_{1}^{k}, \ldots, X_{N}^{k}, V_{1}^{k}, \ldots, V_{N}^{k}\right)$ approximates $y(k h)$. The scheme (34) depends on two parameters $\theta$ and $\mu$. In the special case $K_{n}=0$ (or if $k_{n}=0$ and $\mu=1-\theta$ ), one recovers the classical $\theta$ method [40]. In the general case, (34) corresponds to an additive $\theta$ method where the contact and restoring forces are discretized using different coefficients.

In the sequel, we follow the general procedure described in section 3.1 and consider a modified equation corresponding to the scheme (34) :

$$
\dot{Y}=f(Y)+h F_{1}(Y)+O\left(h^{2}\right),
$$

where the coefficient $F_{1}$ needs to be determined.

Provided $h$ is small enough, system (34) defines $Y_{k+1}$ as an implicit function of $Y_{k}$ and $h$ (this follows from similar arguments as in section A.2). Back substitution in the right-hand side of (34) leads to the explicit reformulation (21), where

$$
\phi(Y, h)=f(Y)+h \phi_{1}(Y)+O\left(h^{2}\right)
$$


and $\phi_{1}$ has to be determined. Let us set $Y=(X, V)$ with $X=\left(X_{1}, \ldots, X_{N}\right), V=\left(V_{1}, \ldots, V_{N}\right)$. Inserting (21) and (36) in (34) and identifying terms of order $h$, we find

$$
\phi_{1}(Y)=\left(\begin{array}{c}
\theta g(X) \\
\varphi(Y)
\end{array}\right)
$$

where $g(X) \in \mathbb{R}^{N}$ is defined in (33) and the components of $\varphi(Y) \in \mathbb{R}^{N}$ are given by

$$
\varphi_{n}(Y)=\frac{1}{m_{n}}\left(-K_{n}(1-\mu) V_{n}+\frac{3}{2} \theta\left(k_{n} \Delta_{(n-1)+}^{1 / 2} \Delta_{n-1}^{\prime}-k_{n+1} \Delta_{n+}^{1 / 2} \Delta_{n}^{\prime}\right)\right),
$$

with $\Delta_{n}=X_{n}-X_{n+1}$ and $\Delta_{n}^{\prime}=V_{n}-V_{n+1}$. From identity (29) and expression (37), we obtain the first order correction term in the modified equation (35):

$$
F_{1}(Y)=\left(\begin{array}{c}
\left(\theta-\frac{1}{2}\right) g(X) \\
\psi(Y)
\end{array}\right)
$$

where the components of $\psi(Y) \in \mathbb{R}^{N}$ are given by

$$
\psi_{n}(Y)=\frac{1}{m_{n}}\left(-K_{n}\left(\frac{1}{2}-\mu\right) V_{n}+\frac{3}{2}\left(\theta-\frac{1}{2}\right)\left(k_{n} \Delta_{(n-1)+}^{1 / 2} \Delta_{n-1}^{\prime}-k_{n+1} \Delta_{n+}^{1 / 2} \Delta_{n}^{\prime}\right)\right) .
$$

As a consequence, the modified equation (35) can be rewritten

$$
\begin{aligned}
\dot{X}_{n}=V_{n} & +h\left(\theta-\frac{1}{2}\right) g_{n}(X)+O\left(h^{2}\right), \\
m_{n} \ddot{X}_{n}+K_{n}\left(h(\theta-\mu) \dot{X}_{n}+X_{n}\right)= & k_{n}\left(\Delta_{(n-1)+}^{3 / 2}+3\left(\theta-\frac{1}{2}\right) h \Delta_{(n-1)+}^{1 / 2} \dot{\Delta}_{n-1}\right) \\
& -k_{n+1}\left(\Delta_{n+}^{3 / 2}+3\left(\theta-\frac{1}{2}\right) h \Delta_{n+}^{1 / 2} \dot{\Delta}_{n}\right)+O\left(h^{2}\right) .
\end{aligned}
$$

With equation (40) at hand, we are now able to qualitatively analyze discretization effects induced by the scheme (34). A local linear damping is present when $\theta>\mu$, but this effect disappears for $\mu=\theta$. The anti-damping that occurs for $\mu>\theta$ corresponds to a numerical instability. The scheme also introduces a contact damping that we now examine in more detail. Setting

$$
\theta=\frac{1}{2}+\frac{\gamma}{2 h}=\mu
$$

in (40) and neglecting $O\left(h^{2}\right)$ terms, one recovers the KK model

$$
m_{n} \ddot{X}_{n}+K_{n} X_{n}=k_{n} \Delta_{(n-1)+}^{3 / 2}+\frac{3}{2} \gamma k_{n} \Delta_{(n-1)+}^{1 / 2} \dot{\Delta}_{n-1}-k_{n+1} \Delta_{n+}^{3 / 2}-\frac{3}{2} \gamma k_{n+1} \Delta_{n+}^{1 / 2} \dot{\Delta}_{n} .
$$

This observation leads us to the concept of tailored numerical dissipation. In this framework, one discretizes the nondissipative model (31) with the dissipative scheme (34)-(41) in order to approximate the dissipative model (42). From the above computations, for $\mu=\theta$ the scheme (34) is consistent at order 2 with the KK model (39)-(42) with damping $\gamma=(2 \theta-1) h$ fixed by (41).

Using this approach, one cannot expect convergence of solutions of (34)-(41) towards solutions of (42) when $\gamma$ is fixed and $h \rightarrow 0$, due to the divergence of the coefficient $\theta$ in (41). However, as we shall see in section 3.5, numerical tests performed for $K_{n}=0$ indicate that the scheme (34) approximates solutions of (42) up to an error $O\left(h^{2}\right)$ when $\gamma=O(h)$ is fixed by (41). The order of approximation is the same for positions and velocities provided one applies the following correction to $V_{n}$ :

$$
\dot{X}_{n}(k h) \approx V_{n}^{k}+\frac{h}{m_{n}}\left(\theta-\frac{1}{2}\right)\left(-K_{n} X_{n}^{k}+k_{n}\left(\Delta_{n-1}^{k}\right)_{+}^{3 / 2}-k_{n+1}\left(\Delta_{n}^{k}\right)_{+}^{3 / 2}\right),
$$

which corresponds to neglecting $O\left(h^{2}\right)$ terms in (39).

Note that in the above computations, some care must be taken concerning the regularity of solutions and the order of the method, due to the limited smoothness of the KK model (see section 2.2). The 
formal second-order consistency that follows from the dissipation condition (41) is actually valid in the absence of contact creation or breaking between the beads (in such regions of the phase space, dynamical equations are infinitely smooth). However, if contact creation or breaking occurs, we show in section A.2 that the consistency error is only $O\left(h^{3 / 2}\right)$. As a consequence, our estimate of the global error is only $O\left(h^{3 / 2}\right)$ instead of $O\left(h^{2}\right)$ (see theorem 1 in section A.2).

The scheme (34)-(41)-(43) will be thereafter denoted as additive $\theta$ method, or simply $\theta$ method in the special case $K_{n}=0$ (this case will be studied in section 4). Note that in the nondissipative case $\gamma=0$, one obtains $\theta=1 / 2$ and one recovers the $\mathrm{CN}$ scheme studied in section 2.2.

Besides the aspects related to convergence, the additive $\theta$ method provides a qualitatively correct approximation of the dynamics of (42) even for relatively large step sizes. In particular, the scheme reproduces an asymptotic synchronisation of the oscillators which originates from the contact damping in (42) and the absence of local damping. In order to illustrate these aspects, we consider a Newton's cradle consisting of $N=10$ identical elements, with contact damping parameter $\gamma=0.3$ (the other parameters are precised in figures 4 and 5). We choose a time step $h=0.5$, which is relatively large considering the collision time $T_{\text {col }}$ between two free beads $\left(T_{\text {col }} \sim 6 h\right)$, but small compared to the period $T_{\text {osc }}$ of in-phase oscillations $\left(T_{\text {osc }} \sim 125 h\right)$. According to the dissipation condition (41), this choice leads us to fix $\theta=0.8$. We consider the impact initial condition (3) with $V_{\text {imp }}=1$.

The dynamics of Newton's cradle is illustrated in figure $4\left(k_{n}=13.115\right)$ and figure $5\left(k_{n}=1\right)$. Each figure compares the reference solution computed by the Matlab ode23s solver for stiff systems (tolerance $10^{-8}$ ) and the approximation obtained with the additive $\theta$ method (system (34) is solved with the fsolve Matlab function with tolerance $10^{-4}$ ). One can observe that both numerical schemes reproduce the same dynamical behavior, characterized by an alternance of short impact phases (where energy is dissipated through contact damping) and slow oscillations (where beads are not in contact, and either the first or last bead oscillates with the highest amplitude), as illustrated by figure 4 . After this transient phase, beads tend to oscillate almost in phase with almost equal amplitudes (figure 5).

From a more quantitative point of view, relatively small differences between the two solutions are visible in figure 4 during the transient phase (with an error close to $7 \%$ on the maximal deflection of the last bead following the initial impact), but discrepancies tend to become negligible when time increases. Comparing the two panels of figure 5, it is hard to see a difference between the two solutions on longer time scales. On the whole time of simulation (which corresponds to almost 48 periods of the linear local oscillators), the error measured with the (rather stringent) supremum norm is 0.155 for displacements and 0.0475 for velocities (dividing by the maximal displacement and velocity, this yields relative errors around $5 \%$ ).

In the sequel, we will introduce a class of two-stage IRK schemes which provide a higher order approximation of the KK model and more accurate results than the $\theta$ method, even for large step sizes.

3.3. Order conditions for two-stage RK schemes with tailored dissipation. We start this section by providing the modified equation for a general two-stage IRK scheme applied to an arbitrary smooth ODE in $\mathbb{R}^{d}$ of the form (20). This problem can be addressed by following the computation scheme described in section 3.1. We summarize below the main steps of the computations which are detailed in [39]. The IRK scheme takes the form [44]

$$
\frac{Y_{k+1}-Y_{k}}{h}=b_{1} k_{1}+\left(1-b_{1}\right) k_{2}
$$

where $k_{1}, k_{2} \in \mathbb{R}^{d}$ are obtained by solving a $2 d$-dimensional nonlinear system

$$
k_{i}=f\left(Y_{k}+h\left(a_{i 1} k_{1}+a_{i 2} k_{2}\right)\right), \quad i=1,2 .
$$

The scheme parameters are $b_{1}$ and the $2 \times 2$ matrix $A=\left(a_{i j}\right)$. By solving (45) for $h$ small enough and defining

$$
\phi\left(Y_{k}, h\right)=b_{1} k_{1}+\left(1-b_{1}\right) k_{2},
$$




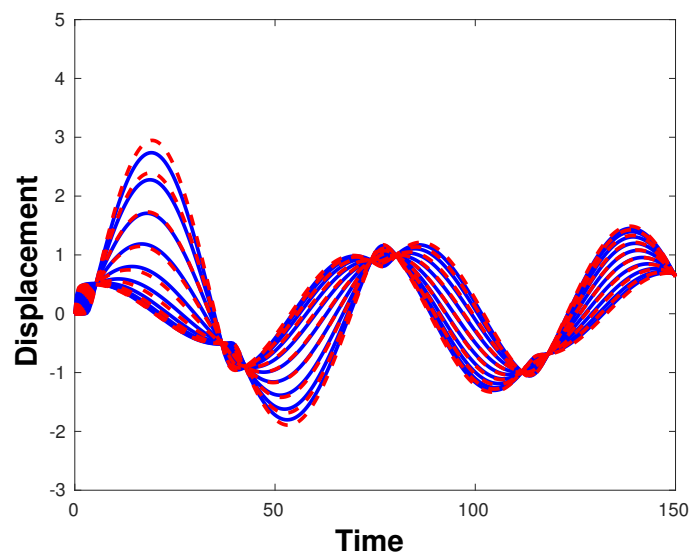

FIGURE 4. Time histories of bead displacements in Newton's cradle $(N=10)$. The blue solid lines represent the reference solution of (42) computed with ode23s. During the slow phases, beads are not in contact and the graphs correspond to beads $n=$ $1, \ldots, N$ from bottom to top. The red dashed lines denote the approximation of the solution by the additive $\theta$ method with $h=0.5$. The system parameters are $m_{n}=1$, $K_{n}=0.01, k_{n}=13.115$ and $\gamma=0.3$.
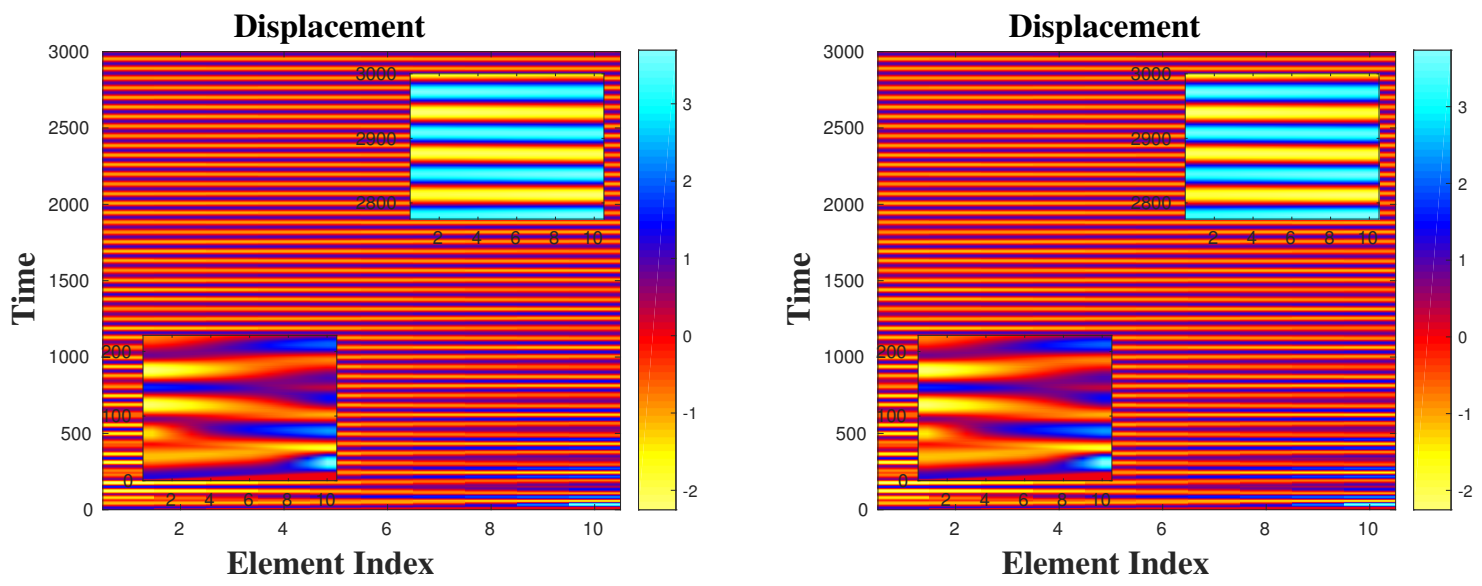

FIGURE 5. Space time diagrams showing bead displacements in Newton's cradle. Parameters are the same as in figure 4 except $k_{n}=1$. The left and right panels display the solutions obtained with ode $23 \mathrm{~s}$ and the additive $\theta$ method, respectively (the two contour plots are almost identical). In each panel, two insets provide a zoom on the solution over long times and during the transient regime preceding synchronization.

the IRK scheme can be reformulated in the equivalent explicit form (21), and we have $\phi(Y, 0)=$ $f(Y)$. The Taylor expansion of $\left(k_{1}, k_{2}\right)$ with respect to $h$ can be computed by a standard identification procedure in system (45), which in turn yields the Taylor expansion of $\phi$ (see [39], Theorem 1.4 p.55 and Table 1.1 p.53).

Following the approach described in section 3.1, we consider the modified equation at the third order relative to the IRK scheme :

$$
\frac{d Y}{d t}=f(Y)+h F_{1}(Y)+h^{2} F_{2}(Y)+O\left(h^{3}\right) .
$$


The coefficients $F_{1}, F_{2}$ are determined in such a way that the IRK scheme and (47) are consistent at order 3, i.e., the consistency error $R_{Y, h}$ defined by (23) is $O\left(h^{3}\right)$. One obtains with the Taylor expansion of $\phi$ and equations (29)-(30) (see [39], section IX.9, formula (9.2) p.369) :

$$
F_{1}=C_{11} f^{[1]}, \quad F_{2}=\frac{1}{2}\left(C_{22}-2 C_{11}\right) L_{f^{[1]}} f+\frac{1}{2}\left(C_{21}-C_{11}\right) f^{(2)},
$$

where $f^{[1]}=L_{f} f, f^{(2)}(y)=D^{2} f(y)(f(y), f(y))\left(D^{2}\right.$ denotes the second derivative $)$ and

$$
\begin{gathered}
C_{11}=b_{1}\left(a_{11}+a_{12}\right)+\left(1-b_{1}\right)\left(a_{21}+a_{22}\right)-\frac{1}{2} \\
C_{21}=b_{1}\left(a_{11}+a_{12}\right)^{2}+\left(1-b_{1}\right)\left(a_{21}+a_{22}\right)^{2}-\frac{1}{3} \\
C_{22}=2 b_{1}\left(a_{11}\left(a_{11}+a_{12}\right)+a_{12}\left(a_{21}+a_{22}\right)\right)+2\left(1-b_{1}\right)\left(a_{21}\left(a_{11}+a_{12}\right)+a_{22}\left(a_{21}+a_{22}\right)\right)-\frac{1}{3} .
\end{gathered}
$$

In the sequel, we address more specifically the case when $d=2 N$ and the right-hand side of (20) reads

$$
f(y)=\left(\begin{array}{c}
v \\
g(x)
\end{array}\right), \quad \forall y=(x, v) \in\left(\mathbb{R}^{N}\right)^{2},
$$

where $g$ denotes a smooth map in $\mathbb{R}^{N}$. Problem (20)-(52) is then equivalent to the second order system

$$
\ddot{x}=g(x) \text {. }
$$

Equation (53) is time-reversible, i.e., it has the invariance $t \rightarrow-t$. Setting $Y=(X, V) \in\left(\mathbb{R}^{N}\right)^{2}$ and using expressions (48) and (52), the modified equation (47) can be rewritten

$$
\begin{gathered}
\dot{X}=V+h C_{11} g(X)+\frac{h^{2}}{2}\left(C_{22}-2 C_{11}\right) D g(X) V+O\left(h^{3}\right), \\
\ddot{X}=g(X)+h 2 C_{11} D g(X) \dot{X}+h^{2}\left(\lambda_{1} L_{g} g(X)+\lambda_{2} D^{2} g(X)(\dot{X}, \dot{X})\right)+O\left(h^{3}\right),
\end{gathered}
$$

with

$$
\lambda_{1}=C_{22}-2 C_{11}-C_{11}^{2}, \quad \lambda_{2}=\frac{1}{2}\left(C_{21}+C_{22}-3 C_{11}\right) .
$$

Thereafter, we follow the approach introduced in section 3.2 on the example of Newton's cradle, and use the scheme (44)-(45) to approximate the following perturbation of (53) :

$$
\ddot{x}=g(x)+\gamma D g(x) \dot{x} .
$$

The parameter $\gamma$ controls the strength of the perturbation that breaks the time-reversibility symmetry in (56). Moreover, if $g=-M^{-1} \nabla \Psi$ for a smooth convex potential $\Psi: \mathbb{R}^{N} \rightarrow \mathbb{R}$ and a positive definite symmetric $N \times N$ matrix $M$, and if one assumes $\gamma>0$, then the energy $H=\frac{1}{2} \dot{x}^{T} M \dot{x}+\Psi(x)$ satisfies

$$
\dot{H}=-\gamma \dot{x}^{T} D^{2} \Psi(x) \dot{x} \leq 0,
$$

i.e., system (56) represents a dissipative perturbation of the conservative system (53).

The modified equation (55) coincides with the perturbed system (56) up to $O\left(h^{2}\right)$ terms under the dissipation condition

$$
C_{11}=\frac{\gamma}{2 h}
$$

Consequently, if $C_{11}$ is fixed and $\gamma=2 C_{11} h$, then the IRK scheme is consistent at order 2 with model (56) supplemented by (54). Furthermore, equations (55) and (56) coincide up to $O\left(h^{3}\right)$ terms under the additional conditions $\lambda_{1}=\lambda_{2}=0$, which can be rewritten as

$$
C_{22}=2 C_{11}+C_{11}^{2}, \quad C_{21}=C_{11}-C_{11}^{2} .
$$

Consequently, the conditions (58)-(59) imply that the IRK scheme is consistent at order 3 with model (56) and equation (54). 
Let us examine the above results more closely in the case of Newton's cradle, which is described by equation (31) in the nondissipative case. In this system we have $M=\operatorname{diag}\left(m_{1}, \ldots, m_{N}\right)$,

$$
\Psi(x)=\frac{1}{2} \sum_{n=1}^{N} K_{n} x_{n}^{2}+\frac{2}{5} \sum_{n=2}^{N} k_{n}\left(x_{n-1}-x_{n}\right)_{+}^{5 / 2}
$$

and the energy reads $H=\Psi(x)+\frac{1}{2} \sum_{n=1}^{N} m_{n} \dot{x}_{n}^{2}$. The modified equation (55) takes the form :

$$
m_{n} \ddot{X}_{n}+K_{n}\left(\gamma \dot{X}_{n}+X_{n}\right)=k_{n}\left(\Delta_{(n-1)+}^{3 / 2}+\frac{3}{2} \gamma \Delta_{(n-1)+}^{1 / 2} \dot{\Delta}_{n-1}\right)-k_{n+1}\left(\Delta_{n+}^{3 / 2}+\frac{3}{2} \gamma \Delta_{n+}^{1 / 2} \dot{\Delta}_{n}\right)+O\left(h^{m}\right),
$$

where $\Delta_{n}=X_{n}-X_{n+1}, m=2$ under condition (58), and $m=3$ if conditions (58)-(59) hold true. Consequently, the modified equation (60) incorporates a contact damping term corresponding to KK dissipation, similarly to equation (42). If $K_{n}=0$, the KK model (4) and equation (60) coincide up to $O\left(h^{m}\right)$ terms. In that case, the KK model can be interpreted as a modified equation at the $m$ th order relative to the IRK scheme.

If $K_{n} \neq 0$, one can notice from equation (60) that the IRK scheme introduces a local damping which is proportional to the contact damping and cannot be tuned independently. In particular, the model (42) cannot be approximated by an equivalent equation within this class of IRK schemes, since this model incorporates contact damping but no local damping. In section 3.2, this issue was solved by introducing an additive $\theta$ method for which local and contact forces were discretized differently (see equation (34)). This scheme belongs to the more general class of additive RK methods [41], which could be used in place of (44)-(45) and would allow us to tune local and contact numerical dissipations independently.

We end this section with some comments on the order of the method and regularity issues. As already noted in section 3.2, the above arguments must be interpreted with caution if the IRK scheme is applied to a situation where contact creation or breaking occurs in a granular chain. In this case, the coefficient of the modified equation (55) at order $h^{j}$ presents a singularity for all $j \geq 2$. In particular, the coefficient $D^{2} g(X)(\dot{X}, \dot{X})$ at order $h^{2}$ reads

$$
\left(D^{2} g(X)(\dot{X}, \dot{X})\right)_{n}=\frac{3}{4 m_{n}}\left(k_{n} H\left(\Delta_{n-1}\right) \Delta_{n-1}^{-1 / 2} \dot{\Delta}_{n-1}^{2}-k_{n+1} H\left(\Delta_{n}\right) \Delta_{n}^{-1 / 2} \dot{\Delta}_{n}^{2}\right),
$$

where $H$ denotes the Heaviside step function. This term is clearly singular when $\Delta_{n} \rightarrow 0^{+}$for some index $n \in\{1, \ldots, N-1\}$, i.e., when the compression between beads $n$ and $n+1$ tends to vanish. We did not attempt to analytically estimate the global error obtained with this class of IRK schemes under conditions (58)-(59). However, for a particular choice of parameters, numerical experiments performed in section 3.5 for $\gamma=O(h)$ indicate $O\left(h^{5 / 2}\right)$ convergence instead of $O\left(h^{3}\right)$.

3.4. An $A$-stable third order IRK scheme with tailored dissipation. In what follows, we construct a two-stage IRK scheme having convenient stability properties and satisfying the order conditions (59), where $C_{11}$ is assumed positive and treated as a parameter. The parameters of the scheme (44)-(45) are searched in the form

$$
\begin{gathered}
b_{1}=\frac{1}{2}+\beta, \\
\left\{\begin{array}{l}
a_{11}=\frac{1}{4}+C_{11}+\alpha, \quad a_{12}=\frac{1}{4}-\frac{\sqrt{3}}{6}-\alpha+\frac{\beta}{\sqrt{3}}, \\
a_{21}=\frac{1}{4}+\frac{\sqrt{3}}{6}+\alpha+\frac{\beta}{\sqrt{3}}, \quad a_{22}=\frac{1}{4}+C_{11}-\alpha,
\end{array}\right.
\end{gathered}
$$

where the coefficients $\alpha=\alpha\left(C_{11}\right)$ and $\beta=\beta\left(C_{11}\right)$ will be determined as functions of $C_{11}$ in order to fulfill the two order conditions (59).

As we shall see, the order conditions lead to $\alpha(0)=\beta(0)=0$, so that the scheme (62)-(63) is a perturbation of the GL scheme (13)-(14)-(15) when $C_{11}$ is small. It is clear that the order conditions (59) are satisfied for $C_{11}=\alpha=\beta=0$, because the GL scheme has order 4, which implies $\mathcal{F}(Y, h)=$ 
$f(Y)+O\left(h^{4}\right)$ in (27). Small values of $C_{11}$ will occur for weakly dissipative systems (where $\gamma$ is small in (57)) due to condition (58) (assuming that the time step $h$ is not too small). Since the GL scheme possesses good stability properties [45] and is symplectic for Hamiltonian systems [44, 39], one can expect the scheme (62)-(63) to possess convenient qualitative properties in the case of weak dissipation.

Let us now determine $\alpha, \beta$ from the two order conditions (59). With the choice (62)-(63), the coefficient $C_{21}$ given by (50) is independent of $\alpha$ and takes the form

$$
C_{21}=-\frac{1}{3} \beta^{2}+C_{11}+C_{11}^{2} \text {. }
$$

Assuming further $\beta \geq 0$, the second order condition $C_{21}=C_{11}-C_{11}^{2}$ yields $\beta=\sqrt{6} C_{11}$. Substituting this value in the expression of $C_{22}$ (equation (51)), one obtains after lengthly but straightforward computations

$$
C_{22}=(2+\sqrt{2}) C_{11}+6 C_{11}^{2}-\frac{2}{\sqrt{3}} \alpha .
$$

The order condition $C_{22}=2 C_{11}+C_{11}^{2}$ leads then to

$$
\alpha=\sqrt{\frac{3}{2}} C_{11}+5 \frac{\sqrt{3}}{2} C_{11}^{2} .
$$

Consequently, we have obtained a family of IRK schemes parameterized by $C_{11} \geq 0$, defined by (64) and

$$
\begin{gathered}
b_{1}=\frac{1}{2}+\sqrt{6} C_{11}, \\
\left\{\begin{array}{l}
a_{11}=\frac{1}{4}+C_{11}+\alpha, \quad a_{12}=\frac{1}{4}-\frac{\sqrt{3}}{6}-\alpha+\sqrt{2} C_{11}, \\
a_{21}=\frac{1}{4}+\frac{\sqrt{3}}{6}+\alpha+\sqrt{2} C_{11}, \quad a_{22}=\frac{1}{4}+C_{11}-\alpha,
\end{array}\right.
\end{gathered}
$$

which satisfies the order conditions (59). The stability of the IRK scheme is studied in section A.1, where we show in particular that the method is $A$-stable for $C_{11} \geq 0$ (see the appendix for the definition of $A$-stability).

From the above computations, in order to approximate the solutions of the perturbed system (56), one can use the IRK scheme (44)-(45) with parameters fixed by the dissipation condition (58) and (64)(65)-(66), applied to the unperturbed system with right-hand side (52). In that case (using the order conditions), the equivalent equation (54)-(55) of the IRK scheme simplifies to

$$
\begin{gathered}
\dot{X}=V+h C_{11} g(X)+\frac{h^{2}}{2} C_{11}^{2} D g(X) V+O\left(h^{3}\right), \\
\ddot{X}=g(X)+\gamma D g(X) \dot{X}+O\left(h^{3}\right),
\end{gathered}
$$

and (68) coincides with (56) up to $O\left(h^{3}\right)$ terms. From equation (67), the following correction must be applied in order to approximate time derivatives up to $O\left(h^{3}\right)$ :

$$
\dot{X}(k h) \approx \dot{X}_{k} \stackrel{\text { def }}{=} V_{k}+h C_{11} g\left(X_{k}\right)+\frac{h^{2}}{2} C_{11}^{2} D g\left(X_{k}\right) V_{k},
$$

where $Y_{k}=\left(X_{k}, V_{k}\right)$ denotes a solution of (44)-(45).

One can notice that for a given initial condition at $t=0$, if $X_{0}=X(0)$ and $D g\left(X_{0}\right) \neq 0$, then the determination of $V_{0}$ requires to solve the following linear system originating from approximation (69):

$$
\left(I+\frac{h^{2}}{2} C_{11}^{2} D g\left(X_{0}\right)\right) V_{0}=\dot{X}(0)-h C_{11} g\left(X_{0}\right) .
$$

Then, with $\left(X_{0}, V_{0}\right)$ at hand, $Y_{k}$ is computed from (44)-(45) by induction, which determines $X_{k} \approx$ $X(k h), V_{k}$, and $\dot{X}_{k} \approx \dot{X}(k h)$ is obtained with (69). Another possibility is to compute the first iterate 
using the rule

$$
V_{0}=\dot{X}(0)-h C_{11} g\left(X_{0}\right)-\frac{h^{2}}{2} C_{11}^{2} D g\left(X_{0}\right) \dot{X}(0),
$$

since identities (70) and (71) coincide up to $O\left(h^{3}\right)$ terms.

In the sequel, this scheme will be applied to the KK model (4). For convenience, we summarize below the different features of the scheme. The coefficients $b_{1}$ and $a_{i j}$ of the two-stage IRK scheme are given by (65) and (66), where $C_{11}$ is related to the KK damping $\gamma$ and time step $h$ through the dissipation condition (58), and $\alpha$ is a function of $C_{11}$ defined in (64).

The scheme defines a sequence of vectors $Y_{k}=\left(X_{1}^{k}, \ldots, X_{N}^{k}, V_{1}^{k}, \ldots, V_{N}^{k}\right)$, where $X_{n}^{k}$ approximates bead positions $x_{n}(k h)$. Bead velocities are approximated according to (69):

$$
\begin{aligned}
& \dot{x}_{n}(k h) \approx V_{n}^{k}+\frac{1}{m_{n}} h C_{11}\left(k_{n}\left(\Delta_{n-1}^{k}\right)_{+}^{3 / 2}-k_{n+1}\left(\Delta_{n}^{k}\right)_{+}^{3 / 2}\right) \\
& +\frac{3}{4 m_{n}} h^{2} C_{11}^{2}\left(k_{n}\left(\Delta_{n-1}^{k}\right)_{+}^{1 / 2}\left(V_{n-1}^{k}-V_{n}^{k}\right)-k_{n+1}\left(\Delta_{n}^{k}\right)_{+}^{1 / 2}\left(V_{n}^{k}-V_{n+1}^{k}\right)\right),
\end{aligned}
$$

where $\Delta_{n}^{k}=X_{n}^{k}-X_{n+1}^{k}$. Moreover, we initialize the IRK scheme with $X_{n}^{0}=x_{n}(0)$ and

$$
\begin{aligned}
& V_{n}^{0}=\dot{x}_{n}(0)+\frac{1}{m_{n}} h C_{11}\left(-k_{n}\left(\Delta_{n-1}^{0}\right)_{+}^{3 / 2}+k_{n+1}\left(\Delta_{n}^{0}\right)_{+}^{3 / 2}\right) \\
& +\frac{3}{4 m_{n}} h^{2} C_{11}^{2}\left(-k_{n}\left(\Delta_{n-1}^{0}\right)_{+}^{1 / 2}\left(\dot{x}_{n-1}(0)-\dot{x}_{n}(0)\right)+k_{n+1}\left(\Delta_{n}^{0}\right)_{+}^{1 / 2}\left(\dot{x}_{n}(0)-\dot{x}_{n+1}(0)\right)\right),
\end{aligned}
$$

following approximation (71).

At each iteration, starting from a known iterate $Y_{k}$, one solves the $4 N$-dimensional nonlinear system (45) (the map $f$ in the right-hand side is the case $K_{n}=0$ of (32)-(33)), and one obtains $Y_{k+1}$ from identity (44).

3.5. Orders of convergence. In section A.2, the convergence of the $\theta$ scheme is proved for the KK model with small $O(h)$ dissipation. For the sake of simplicity, the proof is carried out for an infinite chain of identical beads without local potential, but the analysis could be readily extended to the general case. If $\theta \geq 1 / 2$ is fixed and the dissipation constant is given by $\gamma=(2 \theta-1) h$ (according to (41)), then the $\theta$ scheme approximates bead positions and velocities up to an error $O\left(h^{3 / 2}\right)$ on any bounded time interval (see Theorem 1 in section A.2). This error bound is weaker than the $O\left(h^{2}\right)$ bound that could be expected from the consistency analysis performed in the absence of contact breaking (section $3.2)$.

In what follows, we perform numerical simulations to test the above error estimate for the $\theta$ scheme and to study the convergence of the IRK scheme. We consider the trimer system introduced in section 2.2 and start with the initial condition (19), for which no contact breaking occurs on $\left[0, T_{\max }\right]$ with $T_{\max }=1.5$. The contact damping is set to $\gamma=h$, hence $\theta=1$ and $C_{11}=1 / 2$ according to (41) and (58). In order to study the orders of convergence of the $\theta$ - and IRK schemes, we compare the numerical solutions to the reference solution obtained with the ode45 Matlab solver (relative tolerance $10^{-13}$, absolute tolerance $10^{-15}$ ). The global errors obtained with the $\theta$ method and IRK scheme for different time steps $h$ are shown in the left panel of figure 6 (the global error is the maximal error in displacement and velocity with respect to the reference solution, taken over $n$ and $t \in\left[0, T_{\max }\right]$ ). As expected from the consistency order of these schemes (deduced from the derivation of the equivalent equations in sections 3.2, 3.3 and 3.4), the $\theta$ method converges with order 2 and and the IRK scheme with order 3 in the absence of contact breaking.

In a second step, we consider the initial condition (3) with impact velocity $V_{\mathrm{imp}}=1$. Fixing $T_{\max }=5$, contact creation and breaking occur in the time interval of simulation $\left[0, T_{\max }\right]$. The global errors obtained with the $\theta$ method and IRK scheme are shown in the right panel of figure 6 . We observe that the order of convergence of the $\theta$ method is still equal to 2 , which indicates that the order 
$3 / 2$ obtained in section A.2 is not optimal (at least for this type of initial condition). In addition, we observe that the order of the IRK scheme drops to 2.5. The loss of cubic convergence is consistent with the singular terms (at contact breaking) obtained in the derivation of the modified equation (see (55) and (61)).
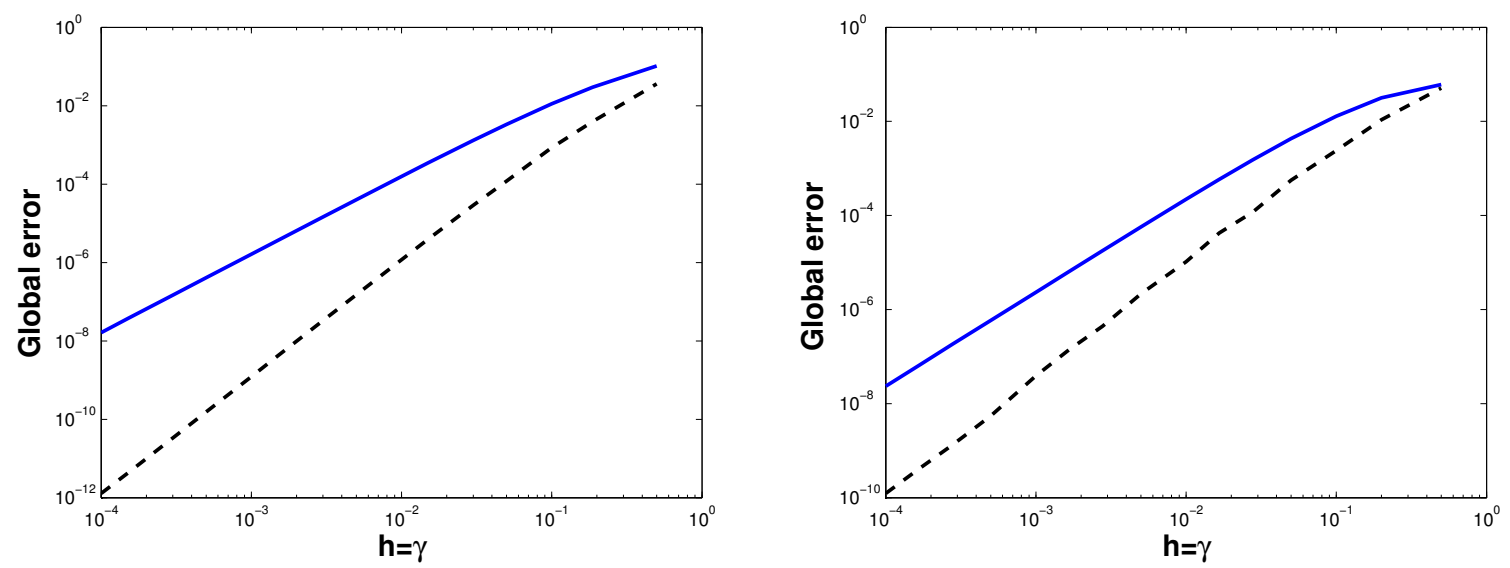

FIGURE 6. Global error of the $\theta$ method (blue line) and IRK scheme (black dashed line) for the initial condition and time interval (19) without contact breaking (left panel), and for the initial condition (3) with impact velocity $V_{\mathrm{imp}}=1$ (right panel).

\section{DisSiPATIVE IMPACTS AND WAVES IN DIMER CHAINS}

In this section, we apply the additive $\theta$ method defined in section 3.2 and the IRK scheme defined in section 3.4 to an impact on a chain consisting of two types of beads. The beads are made of the same material and have alternatively large and small radii. This problem constitutes an interesting test case because the system response displays different wave features depending on the mass ratio between beads. In addition, the effect of the KK contact damping has not been previously analyzed in this context up to our knowledge.

We recall that the $\theta$ and IRK schemes correspond to numerical discretizations of the nondissipative Hertz model, where numerical dissipation is controlled by a single parameter and appropriately tuned in order to match the KK dissipation. For given values of the contact damping constant $\gamma$ and numerical step size $h$, the scheme parameters $\theta$ and $C_{11}$ are fixed by the dissipation conditions (41) for the $\theta$ method and (58) for the IRK scheme. Bead velocities $\dot{x}_{n}$ are retrieved from equations (43) for the $\theta$ scheme and (72) for the IRK scheme. The schemes were implemented both in Matlab and Scilab, and we have used the fsolve functions to solve the implicit equations at each time step.

To evaluate the precision of the $\theta$ and IRK schemes, we will consider reference solutions of the KK model (system (10)-(11) with $\beta=3 / 2$ ) obtained using adaptive ODE solvers with tolerances set to very low values (in the range $10^{-8}-10^{-13}$, depending on the simulation). We use either the ode23s Matlab solver for stiff problems or the ODE solver of Scilab for stiff or non-stiff systems (LSODA ODEPACK solver). In addition, we shall compare the $\theta$ and IRK schemes to the direct discretizations of the KK model introduced in section 2.2. We will evaluate the numerical schemes quantitatively by computing the global error, and qualitatively by studying the robustness of the relevant wave phenomena, both for small and large time steps $h$.

4.1. Solitary waves under dissipation and dispersion effects. We consider equation (4) with $k_{n}=1$, $m_{2 i+1}=1, m_{2 i}=\varepsilon \in(0,1]$, and the impact initial condition (3) with $V_{\mathrm{imp}}=1$. 
The response of the system corresponds to the propagation of a solitary wave in the absence of dissipation (case $\gamma=0$ ). This case was studied in $[12,18]$, where it was shown that the amount of energy dispersion during impact propagation depends on the mass ratio $\varepsilon$. More precisely, for long chains and $\varepsilon \approx 0.59$, one finds a maximal drop in the value of the force transmitted at the end of the chain, while the main pulse is followed by a significant dispersive tail [12]. On the contrary, scattering is minimal for homogeneous chains $(\varepsilon=1)$, leading to a maximal transmitted force. These results have been extended in $[8,18]$ to the case of dissipative chains, using the LZB model $[27,28]$ that accounts for energy loss due to plastic deformations. In what follows, we show that the above phenomenology is preserved by the KK model (4) and the numerical schemes introduced in section 3.

Figure 7 illustrates the propagation of a solitary wave in a dimer chain with $N=25$ elements, for $\varepsilon=0.59$ and $\gamma=0.06$. In the left panel, the blue solid line provides the time history of bead velocities computed from the ode $23 \mathrm{~s}$ scheme with low tolerance. The propagation of a slowly damped solitary wave followed by a sizeable oscillatory tail is clearly visible. When fixing $h=0.1$, the IRK scheme provides a very accurate approximation of the wave profiles (left panel, magenta line). In the right panel, one can notice some discrepancies between the reference solution given by ode $23 \mathrm{~s}$ and the approximation obtained from the IRK scheme with large time step $h=1$. However, the different wave phenomena are still qualitatively well reproduced by the IRK scheme. The $\theta$ scheme requires smaller time steps in order to obtain correct results due to its lower order. For $h=0.1$, the solution obtained with the $\theta$ scheme is very close to the ones displayed in the left panel of figure 7 (data not shown). More quantitative informations are summarized in Table 1.

In the case of an homogeneous chain $(\varepsilon=1)$, the IRK and $\theta$ scheme accurately capture the propagation of a coherent solitary wave and its slow amplitude decay due to contact damping. This is illustrated in figure 8 for $\gamma=0.1$ and $h=0.1$.
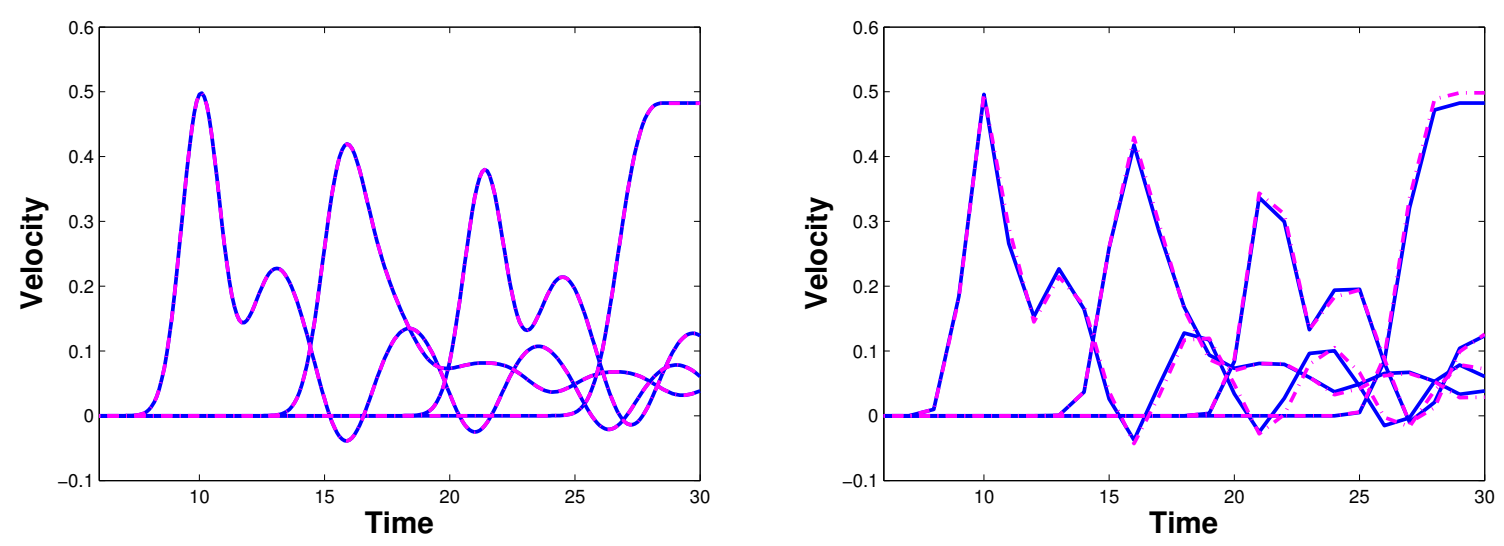

FIGURE 7. Impact propagation in a dimer chain with mass ratio $\varepsilon=0.59, \gamma=0.06$ and $N=25$ elements. The graphs display the time evolution of the velocities of beads number 10, 15, 20 and 25 (from left to right). The blue solid line corresponds to the reference solution obtained with ode23s (tolerance $10^{-13}$ ) and the magenta dash-dot line show the IRK approximation. Left and right panels correspond to $h=0.1$ and $h=1$, respectively.

Figure 9 illustrates the effect of mass ratio on the ejection velocity of the last bead, computed with different schemes and time steps for the same parameters as in figure 7. The results of the IRK and $\theta$ schemes with $h=0.1$ are very close to the reference solution and small discrepancies appear for the IRK scheme with $h=1$, although the graph of the ejection velocity remains qualitatively correct. A shallow local minimum of the ejection velocity is clearly visible close to $\epsilon=0.6$, and is linked with 
GUILLAUME JAMES $^{1,2}$, KIRILL VOROTNIKOV $^{1,2}$, BERNARD BROGLIATO $^{1,2}$

\begin{tabular}{|l|l|l|l|}
\hline Method and time step & $\begin{array}{l}\text { Maximal error } \\
\text { in displacement }\end{array}$ & $\begin{array}{l}\text { Maximal error } \\
\text { in velocity }\end{array}$ & $\begin{array}{l}\text { Execution } \\
\text { time [s] }\end{array}$ \\
\hline IRK scheme $h=1$ & 0.0832 & 0.0447 & 0.18 \\
\hline IRK scheme $h=0.1$ & 0.0033 & $6.1127 \mathrm{e}-04$ & 1.8 \\
\hline$\theta$ scheme $h=0.1$ & 0.0217 & 0.0121 & 0.53 \\
\hline
\end{tabular}

TABLE 1. Numerical errors and execution times for the simulation of the impact propagation described in figure 7 (dimer chain with $N=25$ elements), for different numerical schemes and time steps. Errors are computed in supremum norm, with $1 \leq$ $n \leq N$ and $t \in[0,30]$ (the supremum norm of the reference solution is approximately 2.27 for displacements and 1 for velocities). Timings were measured on a Intel Core i7 processor ( $3 \mathrm{GHz}, 16 \mathrm{~GB}$ RAM) with the numerical algorithms implemented in Scilab.

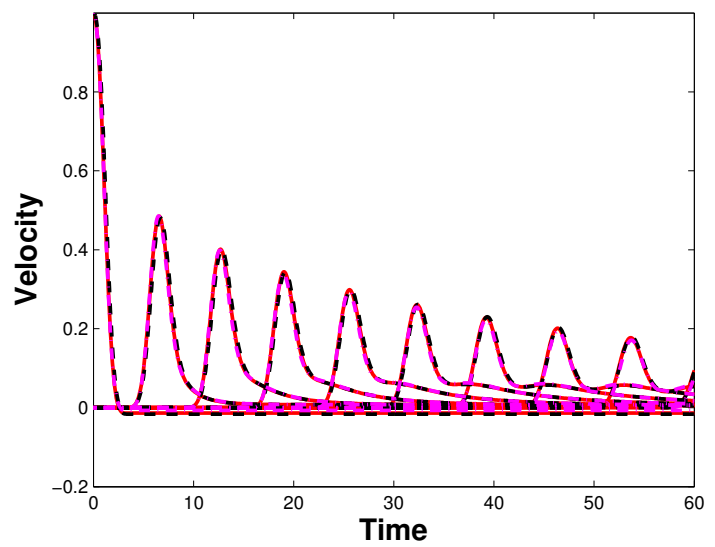

FIGURE 8. Impact propagation in an homogeneous chain with $\gamma=0.1$ and $N=50$ elements. The graphs display the time evolution of the velocities of beads number $1+5 i(0 \leq i \leq 9)$ from left to right. The red solid line corresponds to the reference solution obtained with ode23s (tolerance $10^{-8}$ ), the black dashed line to the $\theta$ scheme, and the magenta dash-dot line to the IRK scheme, with $h=0.1$ for both schemes.

the energy transferred to the oscillatory tail of the solitary wave, similarly to the results reported in $[12,8,18]$.

In summary, the above computations indicate that the concept of tailored numerical dissipation implemented through the $\theta$ and IRK schemes correctly describe impact propagation in dimer chains, even for relatively large step sizes $h$ (in the range $0.1-1$, depending on the scheme).

4.2. Numerical dissipation vs direct discretization. Let us now compare the $\theta$ and IRK schemes to the direct discretizations of the KK model introduced in section 2.2, based on the CN and GL schemes. We recall that in the absence of contact creation or breaking, dynamical equations are perfectly smooth (i.e., the right-hand side is analytic) and thus the latter schemes have orders 2 and 4 , respectively. Impacts lead to order reduction, but this phenomenon can be attenuated to some extent by a regularizing change of variables, switching from the usual discretizations (12) and (13)-(14) to the new ones (16) and (17)-(18).

Tests are performed for a dimer chain with $N=25$ elements and $\varepsilon=0.59$. For given step sizes, we compare the global errors (uniform distance to the reference solution) of the IRK and GL schemes on one hand, and those of the $\theta$ and $\mathrm{CN}$ schemes on the other hand. Note that in each group, the three 


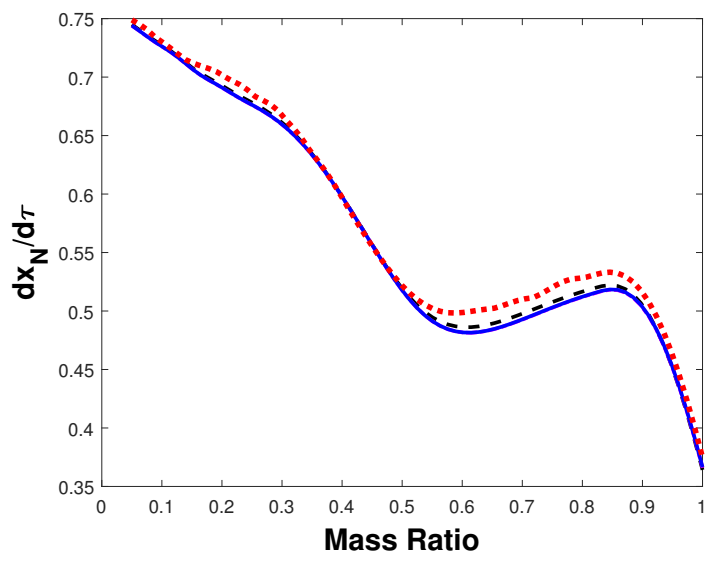

FIGURE 9. Velocity of ejection of the last bead in a dimer chain, plotted as a function of mass ratio $\epsilon$ (the chain has $N=25$ elements and the impact velocity is equal to unity). The contact damping constant is $\gamma=0.06$. The blue solid line shows the reference solution calculated by ode $23 \mathrm{~s}$ (tolerance $10^{-8}$ ), the black dashed line corresponds to the $\theta$ scheme with $h=0.1$, the magenta dash-dot line to the IRK scheme with $h=0.1$ (this curve almost perfectly coincides with the reference solution), and the red dotted line to the IRK scheme with $h=1$. The final velocity of the $N$ th bead is evaluated at $t=30$ (beyond this time the bead performs a free flight).

schemes lead to similar execution times, and at each iteration the $\theta$ and $\mathrm{CN}$ schemes are computationally less expensive than the IRK and GL schemes.

We start by comparing the IRK and GL schemes and first consider a large time step $h=0.5$. The errors resulting from each scheme are given in the left panel of figure 10 for different values of $\gamma \in[0,0.1]$. The smallest error is obtained with the IRK scheme for $\gamma \in[0.007,0.057]$ and with the GL scheme (13)-(14) for $\gamma \in[0.062,0.1]$. Moreover, the errors converge to the same value for the three schemes when $\gamma \rightarrow 0$ because the schemes coincide for $\gamma=0$.

The situation is different for smaller time steps, as illustrated in figure 10 (right panel) for $h=$ 0.05. The error obtained with the IRK scheme is the largest when $\gamma$ lies above 0.013 , and it increases significantly with $\gamma$ in comparison to the GL schemes. This is linked with the fact that the IRK scheme does not converge towards the solution of the KK model when $\gamma>0$ is fixed and $h \rightarrow 0$ (figure 6 suggests convergence for $\gamma=O(h)$ as in Theorem 1), but does converge for $\gamma=0$ and $h \rightarrow 0$ (since it coincides with the GL scheme). In addition, we notice that (17)-(18) provides better results than (13)-(14) for $\gamma$ in some bounded interval away from 0 and $h$ small enough. This is due to the higher order of (17)-(18) when contact creation or breaking occurs.

The case of the $\theta$ and $\mathrm{CN}$ schemes is addressed in figure 11. For a rather large time step $h=0.25$ (left panel), the largest error is produced by the CN scheme (12) (based on the less regular ODE) when $\gamma \in[0.11,0.178]$ and by the $\theta$ method outside this interval. There are no values of $\gamma$ for which the $\theta$ scheme yields the smallest error, but for $\gamma$ below 0.1 the three schemes lead to rather similar errors. The CN scheme (16) provides the best results when $\gamma$ is large enough, above 0.08 for this time step. When $h$ is further decreased, this scheme becomes more advantageous than (12), which suffers from order reduction in the case of impacts. This is illustrated in figure 11 (right panel) for $h=0.01$, where the $\mathrm{CN}$ scheme (16) yields the lowest error for $\gamma$ above 0.05. For the $\theta$ scheme, the error increases rapidly when $\gamma$ departs from 0 and $h$ is small, for the same reason as previously explained for the IRK scheme. 

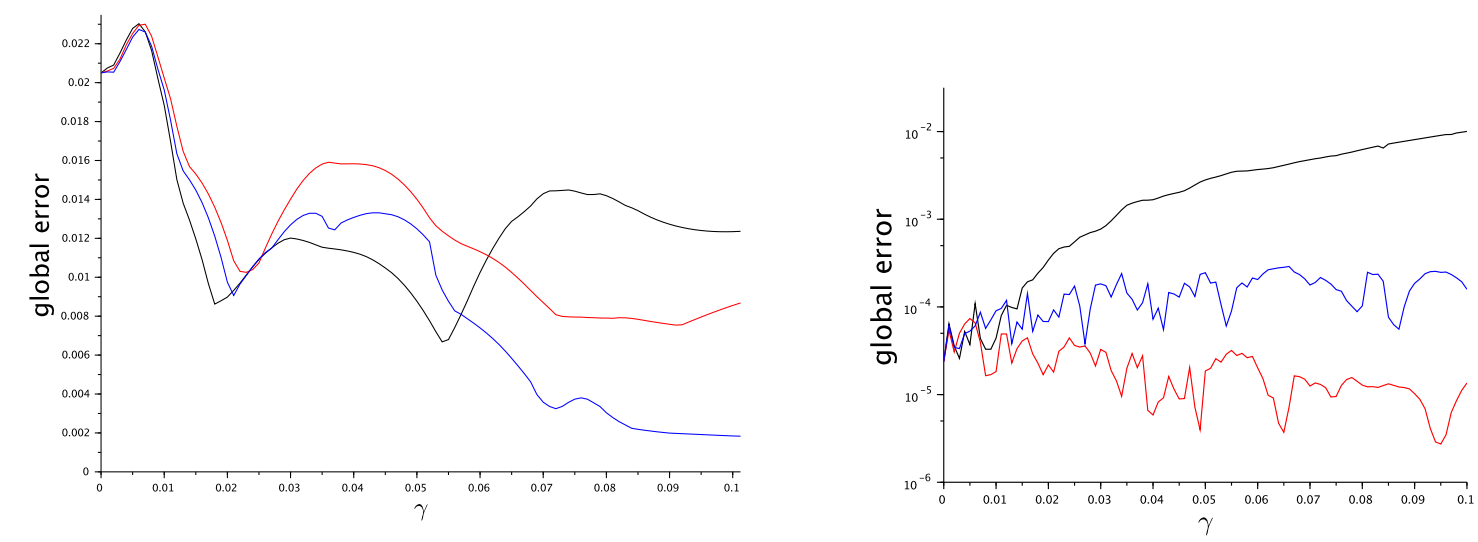

FIGURE 10. Global error resulting from different schemes for an impact propagation in a dimer chain $(\varepsilon=0.59, N=25)$. The black curve corresponds to the IRK scheme, the red curve to the GL scheme (17)-(18), and the blue curve to the GL scheme (13)(14) based on the less regular ODE. The left panel corresponds to a large step size $h=0.5$, while the right panel corresponds to $h=0.05$. Errors are computed in supremum norm (with $1 \leq n \leq N$ and $t \in[0,30]$ ) for different values of the damping constant $\gamma$ (note the semi-logarithmic scale in the right panel). The reference solutions used to evaluate the global error have been computed using the standard ODE solver of Scilab, with relative and absolute error tolerances set to $10^{-13}$ and $10^{-15}$, respectively (the supremum norm of the reference solution decays from approximately 2.94 to 2.06 when $\gamma$ is increased in $[0,0.1]$ ).

As a conclusion, the above numerical results indicate that the best approach for the simulation of impacts in granular chains depends on the range of step sizes considered and the strength of contact damping. In order to obtain more precise results when the time step is small, it is preferable to use a direct discretization of the KK model written in the regularized form (10)-(11), as illustrated in this work with the GL and CN schemes. However, for large time steps and $\gamma$ not too large, the $\theta$ and IRK schemes based on tailored numerical dissipation are competitive with the direct discretizations, and the IRK scheme is even more precise than the GL scheme in some physical range of damping constants $\gamma$.

\section{CONCLUSION AND PERSPECTIVES}

In this work, we have used the technique of modified equations to construct time-integration schemes matching numerical dissipation with physical dissipation at different consistency orders. This approach was applied to an additive $\theta$ method and general two-stage IRK schemes, and allowed us to simulate impacts in dissipative granular chains without explicitly including the nonsmooth viscoelastic force of the KK model. For dimer chains, we have shown that an IRK scheme with KK numerical dissipation provides accurate results for relative large step sizes and compares well with direct discretization of the KK model by the GL scheme. In the above study, particular attention has been paid to the lack of regularity of the KK model. We have numerically studied associated order reduction phenomena, introduced appropriate variables for more accurate time-integration, and provided in section A.2 a convergence proof for the $\theta$ method adapted to the non Lipschitz continuous contact forces.

The above numerical methods will be further improved in several directions. The formulation and resolution of the implicit equations (34) and (45) can be significantly optimized, e.g. by following the line of [49] (chapter 5) and by exploiting the band structure of the dynamical equations. This will 

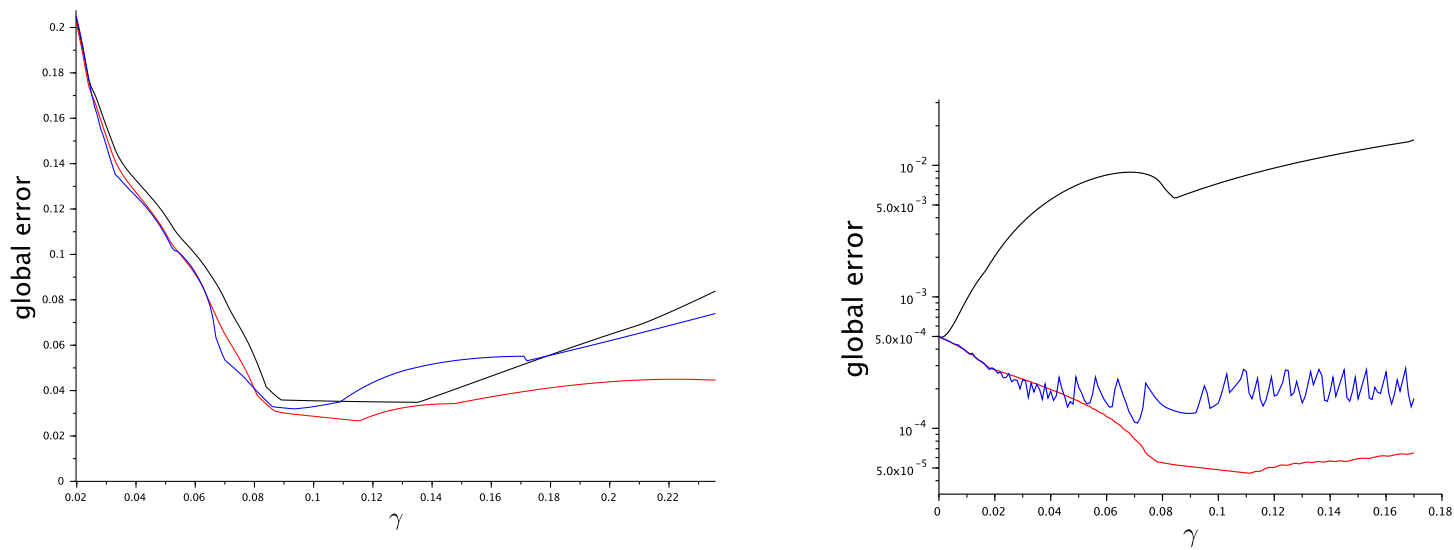

FIGURE 11. Global error resulting from different schemes for an impact propagation in a dimer chain, for the same parameters as in figure 10. The black curve corresponds to the $\theta$ scheme, the red curve to the $\mathrm{CN}$ scheme (16), and the blue curve to the $\mathrm{CN}$ scheme (12) based on the less regular ODE. The left and right panels correspond to $h=0.25$ and $h=0.01$, respectively (note the semi-logarithmic scale in the right panel).

allow for efficient simulations of much larger systems of particles, such as those describing two- or three-dimensional granular crystals (see e.g. [7] and references therein) or a granular layer in contact with a continuum elastic medium [2]. The introduction of adaptive piecewise-constant time steps will also bring important improvements by increasing precision and reducing simulation times.

From a theoretical point of view, it would be interesting to obtain a sharper error bound for the $\theta$ method with KK dissipation and to generalize the convergence analysis to other IRK schemes, including the third order IRK method introduced in section 3.4. Moreover, applying our approach to nonautonomous systems constitutes an additional meaningful extension. On another front, the approximation of unilateral compliant models involving linear spring-dashpots constitutes another interesting problem, where additional difficulties may arise from the discontinuity of dissipative forces.

Another relevant problem concerns the approximation of more general types of dissipative effects. Additive RK schemes [41] could be used to match an arbitrary sum of dissipative forces, just as we have used the additive $\theta$ method to approximate the local and contact dissipations in Newton's cradle. In addition, variable stepsizes [50] may allow us to approximate a wider class of dissipative forces using numerical dissipation, such as the case $\beta \neq 3 / 2$ in (1). If other types of dissipation are present which can hardly be incorporated through numerical dissipation, such as those arising from Coulomb friction and plasticity, one could then consider hybrid discretizations combining numerical dissipation with explicitly incorporated dissipative terms.

\section{ACKNOWLEDGMENTS}

G.J. thanks the PAZI Fund (Israel Atomic Energy Commission) (Grant No. 263/15) for financial support. The authors are grateful to V. Acary, F. Bourrier and A. Vieira for stimulating discussions.

\section{APPENDiX A. STABILITY AND CONVERGENCE ANALYSIS}

A.1. $A$-stability of the two-stage IRK scheme. In this section we analyze the stability of the twostage IRK schemes (44)-(45) parameterized by $C_{11}>0$, with coefficients $a_{i, j}$ and $b_{1}$ defined in (64)(65)-(66). For simplicity we omit the case $C_{11}=0$ which corresponds to the GL scheme, for which 
$A$-stability is already established [45]. In what follows we assume some familiarity with standard stability analysis (see e.g. [45], sections IV.2-3).

We discretize the Dahlquist test equation $\dot{y}=\lambda y$, where $y(t) \in \mathbb{C}$ and $\lambda \in \mathbb{C}$ is a parameter, and therefore consider iteration (44)-(45) with $f(y)=\lambda y$. The numerical solution takes the form $Y_{k}=(R(h \lambda))^{k} Y_{0}$, where $R$ is called the stability function of the method. It consists of a rational function $R=\frac{P}{Q}$ which takes the form [45]

$$
P(z)=\operatorname{det}\left(I-z A+z \mathbb{1} b^{T}\right), \quad Q(z)=\operatorname{det}(I-z A),
$$

where $A=\left(a_{i j}\right), b^{T}=\left(b_{1}, 1-b_{1}\right), \mathbb{1}=(1,1)^{T}$. Using the coefficients defined in (64)-(65)-(66), one obtains after lengthy but straightforward computations

$$
P(z)=1+\left(\frac{1}{2}-2 C_{11}\right) z+a^{-} z^{2}, \quad Q(z)=1-\left(\frac{1}{2}+2 C_{11}\right) z+a^{+} z^{2},
$$

where $a^{ \pm}=\frac{1}{12} \pm \frac{1}{2} C_{11}+\frac{3}{2} C_{11}^{2}$.

In the sequel, we check that the IRK scheme is $A$-stable [45], i.e., we have $|R(z)| \leq 1$ for $\operatorname{Re}(z) \leq 0$. More precisely, we will show that

$$
|R(z)|<1 \text { when } \operatorname{Re}(z) \leq 0 \text { and } z \neq 0 .
$$

Therefore, if the equilibrium $y=0$ of the Dahlquist equation is asymptotically stable (case $\operatorname{Re}(\lambda)<0)$, then this property remains true for the fixed point $Y^{k}=0$ for all step size $h>0$. Similarly, if $\lambda \neq 0$ lies on the imaginary axis (case when $y=0$ is Lyapunov stable), then $Y^{k}=0$ is asymptotically stable for all step sizes, meaning that the scheme is dissipative. This differs from the case $C_{11}=0$ and $\lambda \in i \mathbb{R}$, in which $Y^{k}=0$ is only Lyapunov stable.

We start by checking (76) on the imaginary axis. For all $\omega \in \mathbb{R} \backslash\{0\}$, one has

$$
|Q(i \omega)|^{2}-|P(i \omega)|^{2}=C_{11} \omega^{2}\left(2+\omega^{2}\left(\frac{1}{6}+3 C_{11}^{2}\right)\right)>0
$$

and thus $|R(i \omega)|<1$. In addition, one can check that $Q$ has two conjugate roots with positive real parts whenever $C_{11}$ is nonnegative, and thus $R(z)$ is analytic in the left complex plane $\operatorname{Re}(z) \leq 0$. Consequently, property (76) follows from the maximum principle and the bound $|R(i \omega)|<1$.

We close this section by observing that

$$
\lim _{|z| \rightarrow+\infty}|R(z)|=r, \quad \text { with } r=\frac{a^{-}}{a^{+}}<1 .
$$

This implies that the domain of instability where $|R(z)|>1$ is bounded. Moreover, if $C_{11}$ is either large or close to 0 , then $r$ is close to unity and stiff components of linear ODE (corresponding to large eigenvalues $\lambda$ ) are only slowly damped with the IRK scheme. In contrast, for $C_{11}=\frac{1}{3 \sqrt{2}} \approx 0.236, r$ attains a global minimum $r_{\min }=\frac{\sqrt{2}-1}{\sqrt{2}+1} \approx 0.17$ far from unity.

A.2. Convergence of the $\theta$ method with Kuwabara-Kono numerical dissipation. The aim of this section is to establish the convergence of the $\theta$ method (34)-(43) towards the KK model with dissipation constant given by (41) (some familiarity with the convergence of one-step methods [39, 44, 47] is assumed). For simplicity we consider an infinite chain of identical beads without local restoring forces (i.e., with $K_{n}=0$ and $m_{n}, k_{n}$ constant in (31) and (42)). The KK model reads after suitable rescaling :

$$
\ddot{x}=\delta^{-}\left(1+\tilde{\gamma} \frac{d}{d t}\right) F_{\mathrm{H}}\left(\delta^{+} x\right),
$$

where $x(t)=\left(x_{n}(t)\right)_{n \in \mathbb{Z}}$ describes bead positions, $\left(\delta^{ \pm} x\right)_{n}= \pm\left(x_{n \pm 1}-x_{n}\right)$ denote the usual forward and backward differences, $F_{\mathrm{H}}(r)=-(-r)_{+}^{3 / 2}$ the Hertz force and $\tilde{\gamma} \geq 0$ the nondimensional 
dissipation constant. We consider solutions of $(77)$ in $\ell_{2}(\mathbb{Z})$ (the Hilbert space of real square summable sequences). Since $F_{\mathrm{H}}$ defines a $C^{1}$ map in $\ell_{2}(\mathbb{Z})$ and the energy

$$
H(t)=\frac{1}{2}\|\dot{x}(t)\|_{2}^{2}+\frac{2}{5} \sum_{n \in \mathbb{Z}}\left(x_{n}(t)-x_{n+1}(t)\right)_{+}^{5 / 2}
$$

is nonincreasing along the solutions of (77), one obtains a smooth flow in $\ell_{2}(\mathbb{Z})^{2}$ for $(x(t), \dot{x}(t)-$ $\left.\tilde{\gamma} \delta^{-} F_{\mathrm{H}}\left(\delta^{+} x(t)\right)\right)$ which is globally defined for $t \geq 0$.

Now let us consider the usual $\theta$ scheme for the nondissipative Hertz model :

$$
\frac{Y_{k+1}-Y_{k}}{h}=\theta f\left(Y_{k+1}\right)+(1-\theta) f\left(Y_{k}\right)
$$

where $Y_{k}=\left(X_{k}, V_{k}\right) \in \ell_{2}(\mathbb{Z})^{2}$ for all $k \geq 0, h$ denotes a small step size and $f(x, \dot{x})=\left(\dot{x}, \delta^{-} F_{\mathrm{H}}\left(\delta^{+} x\right)\right)$. We shall prove the following :

Theorem 1. Fix $\theta \geq 1 / 2, H_{0} \geq 0$ and $T>0$. There exist $M>0$ and $h_{\max } \in(0, T)$ such that the following properties hold true for all $h \in\left(0, h_{\max }\right)$, for the dissipation constant of (77) given by

$$
\tilde{\gamma}=(2 \theta-1) h,
$$

and for any solution $x$ of $(77)$ in $\ell_{2}(\mathbb{Z})$ satisfying $H(0)=H_{0}$ :

i) There exists a unique finite sequence $\left(Y_{k}\right)_{0 \leq k \leq T / h} \subset \ell_{2}(\mathbb{Z})^{2}$ solution of (78) for all $k \in\left[0, \frac{T}{h}-1\right]$ and denoted by $Y_{k}=\left(X_{k}, V_{k}\right)$, satisfying the initial condition

$$
Y_{0}=\left(x(0), \dot{x}(0)+\left(\frac{1}{2}-\theta\right) h \delta^{-} F_{\mathrm{H}}\left(\delta^{+} x(0)\right)\right)
$$

and such that $\max _{k h \in[0, T]}\left\|\left(-\delta^{+} X_{k}\right)_{+}\right\|_{\infty} \leq 2\left(5 H_{0} / 2\right)^{2 / 5}$.

ii) The sequence $\left(Y_{k}\right)$ approximates the solution $x$ on $[0, T]$ with :

$$
\begin{gathered}
\max _{k h \in[0, T]}\left\|x(k h)-X_{k}\right\|_{2} \leq M h^{3 / 2}, \\
\max _{k h \in[0, T]}\left\|\dot{x}(k h)-\dot{X}_{k}\right\|_{2} \leq M h^{3 / 2}
\end{gathered}
$$

and $\dot{X}_{k} \stackrel{\text { def }}{=} V_{k}+\left(\theta-\frac{1}{2}\right) h \delta^{-} F_{\mathrm{H}}\left(\delta^{+} X_{k}\right)$.

Proof : as shown in section 3.2, the modified equation corresponding to (78) reads

$$
\frac{d Y}{d t}=f(Y)+\left(\theta-\frac{1}{2}\right) h D f(Y) f(Y)+O\left(h^{2}\right) .
$$

The scheme (78) is consistent with (82) at order 2 in the absence of contact breaking, i.e., whenever $\delta^{+} x(t)<0$. We recall that (82) takes the same form as the KK model with small dissipation. More precisely, setting $y=(x, v)$ with

$$
v=\dot{x}-\frac{\tilde{\gamma}}{2} \delta^{-} F_{\mathrm{H}}\left(\delta^{+} x\right)
$$

equation (77) can be rewritten

$$
\frac{d y}{d t}=f(y)+\frac{\tilde{\gamma}}{2} D f(y) f(y)+\frac{\tilde{\gamma}^{2}}{4} \rho(y)
$$

with $\rho(y)=\delta^{-}\left(0, F_{\mathrm{H}}^{\prime}\left(\delta^{+} x\right) \delta^{2} F_{\mathrm{H}}\left(\delta^{+} x\right)\right)$ and $\delta^{2}=\delta^{+} \delta^{-}$. With the choice (79) of dissipation constant in (84), equations (82) and (84) coincide at leading order in $h$, and thus (78) is consistent with the KK model (84) at order 2 in the absence of contact breaking. 
The next step is to estimate the order of consistency of (78) with (84) including the case of contact breakings. Given an arbitrary solution $y=(x, v)$ of $(84)$, we consider the residual

$$
R(t)=\frac{y(t+h)-y(t)}{h}-\theta f(y(t+h))-(1-\theta) f(y(t))
$$

which has the same order in $h$ as the consistency error (23). The following identity can be derived from (79) and (84) after lengthy algebraic manipulations :

$$
R(t)=\frac{h}{2} \iint_{[0,1]^{2}}(\dot{f}(t+s h)-\dot{f}(t+r h))(r-s) d s d r+\frac{\tilde{\gamma}^{2}}{4} \int_{0}^{1} Q[y(t+s h)] d s,
$$

where $Q(y)=\left(1-\frac{\tilde{\gamma}}{2} D f(y)\right) \rho(y)-D f(y) D f(y) f(y)$ and

$$
\dot{f}(t)=\frac{d}{d t} f(y(t))=\delta^{-}\left[F_{\mathrm{H}}\left(\delta^{+} x\right)\left(\begin{array}{l}
1 \\
0
\end{array}\right)+F_{\mathrm{H}}^{\prime}\left(\delta^{+} x\right) \delta^{+} \dot{x}\left(\begin{array}{c}
\tilde{\gamma} \\
1 \\
1
\end{array}\right)\right](t) .
$$

In the subsequent estimates, we assume $t, \tau \geq 0$ and $h \in(0,1)$. For notational simplicity, we use a unique symbol $C$ to denote multiplicative constants depending solely on $\theta$ and on the initial energy $H(0)$. Since $H(t) \leq H(0)$, velocities $\dot{x}_{n}(t)$ are bounded, absolute displacements $\left|x_{n}(t)\right|$ grow at most linearly in time, and contact forces $F_{\mathrm{H}}\left(x_{n+1}(t)-x_{n}(t)\right)$ are bounded. Using these properties, one can show that $\|Q(y(t))\|_{2} \leq C$ and

$$
\left\|F_{\mathrm{H}}\left(\delta^{+} x(t)\right)-F_{\mathrm{H}}\left(\delta^{+} x(\tau)\right)\right\|_{2} \leq C|t-\tau|
$$

from the mean value inequality. Proceeding in the same way, and using the estimate

$$
\forall a, b \in \mathbb{R}, \quad\left|F_{\mathrm{H}}^{\prime}(a)-F_{\mathrm{H}}^{\prime}(b)\right| \leq \frac{3}{2}|a-b|^{1 / 2}
$$

and the bound $\|\ddot{x}(t)\|_{2} \leq C$ derived from (77), we get

$$
\begin{aligned}
\left\|F_{\mathrm{H}}^{\prime}\left(\delta^{+} x(t)\right) \delta^{+} \dot{x}(t)-F_{\mathrm{H}}^{\prime}\left(\delta^{+} x(\tau)\right) \delta^{+} \dot{x}(\tau)\right\|_{2} & \leq C\left(\|x(t)-x(\tau)\|_{2}^{1 / 2}+\|\dot{x}(t)-\dot{x}(\tau)\|_{2}\right) \\
& \leq C\left(|t-\tau|^{1 / 2}+|t-\tau|\right) .
\end{aligned}
$$

We note in passing that the fractional exponent $1 / 2$ accounts for the possibility of contact breaking. Substitution of the above estimates in (85) leads finally to the following bound for the residual :

$$
\|R(t)\|_{2} \leq C h^{3 / 2}
$$

for all $t \geq 0$ and $h \in(0,1)$.

Having checked the consistency of (78) with (84) through estimate (86), we are now in a position to prove the $O\left(h^{3 / 2}\right)$ convergence of the $\theta$ method towards the KK model.

We begin by modifying $f$ with a cut-off in order to recover the classical setting of a globally Lipschitz continuous map. For this purpose we define $r_{0}=2(5 H(0) / 2)^{2 / 5}$ and denote by $P$ the unique $C^{1}$ function such that $P(r)=r_{+}^{3 / 2}$ for $r \leq r_{0}$ with $P$ affine on $\left[r_{0},+\infty\right)$. We denote similarly $\tilde{F}_{\mathrm{H}}(r)=$ $-P(-r)$, so that $\tilde{f}(x, \dot{x})=\left(\dot{x}, \delta^{-} \tilde{F}_{\mathrm{H}}\left(\delta^{+} x\right)\right)$ defines a Lipschitz continuous map in $\ell_{2}(\mathbb{Z})^{2}$, with constant $L$ depending solely on $H(0)$. From $H(t) \leq H(0)$ it follows that $\left\|\left(-\delta^{+} x(t)\right)_{+}\right\|_{\infty} \leq \frac{r_{0}}{2}$ and thus $f(y(t))=\tilde{f}(y(t))$.

Now we look for a solution $Y_{k}$ of the modified $\theta$ scheme (78) with $f$ replaced by $\tilde{f}$, for the initial condition $Y_{0}=y(0)$. We introduce the error variable $e_{k}=Y_{k}-y(k h)$ which satisfies

$$
\begin{aligned}
e_{k+1}= & e_{k}+h \theta\left(\tilde{f}\left[y((k+1) h)+e_{k+1}\right]-\tilde{f}[y((k+1) h)]\right) \\
& +h(1-\theta)\left(\tilde{f}\left[y(k h)+e_{k}\right]-\tilde{f}[y(k h)]\right)-h R(k h) .
\end{aligned}
$$


This defines a fixed point problem for $e_{k+1}$ in $\ell_{2}(\mathbb{Z})^{2}$ which admits a unique solution when $h<(\theta L)^{-1}$ (this follows from the contraction mapping theorem), hence the sequence $\left(e_{k}\right)_{k \geq 0}$ is uniquely defined by induction. Furthermore, we find

$$
\left\|e_{k+1}\right\|_{2} \leq\left\|e_{k}\right\|_{2}(1+h b L)+h b\|R(k h)\|_{2}
$$

with $b=(1-h \theta L)^{-1}$. Assuming further $h \leq(\theta L)^{-1} / 2$ and $k h \leq T$ for some fixed $T>0$, we then obtain from a discrete Gronwall inequality

$$
\left\|e_{k}\right\|_{2} \leq e^{2 L T} 2 h \sum_{j=0}^{k-1}\|R(j h)\|_{2} .
$$

Consequently, the consistency estimate (86) yields the global error estimate

$$
\max _{k h \in[0, T]}\left\|e_{k}\right\|_{2} \leq C T e^{2 L T} h^{3 / 2} .
$$

Coming back to the numerical solution $Y_{k}=y(k h)+e_{k}=\left(X_{k}, V_{k}\right)$, we get

$$
\max _{k h \in[0, T]}\left\|\left(-\delta^{+} X_{k}\right)_{+}\right\|_{\infty} \leq \frac{r_{0}}{2}+2 \max _{k h \in[0, T]}\left\|e_{k}\right\|_{2} \leq r_{0}
$$

provided $h<h_{\max }$, with $h_{\max }$ depending solely on $\theta, H(0)$ and $T$. In that case, $Y_{k}$ defines a solution of (78) whenever $(k+1) h \leq T$, since $f\left(Y_{k+1}\right)=\tilde{f}\left(Y_{k+1}\right)$ and $f\left(Y_{k}\right)=\tilde{f}\left(Y_{k}\right)$. In the same way, $Y_{k}$ is the unique solution of (78) such that $Y_{0}=y(0)$ and $\max _{k h \in[0, T]}\left\|\left(-\delta^{+} X_{k}\right)_{+}\right\|_{\infty} \leq r_{0}$.

Estimate (88) yields the error bound (80) on bead positions, where $M$ denotes a constant depending solely on $\theta, H(0)$ and $T$. Moreover, the error bound (81) on velocities follows from (79), (83) and (88). This completes the proof of Theorem 1.

\section{REFERENCES}

[1] Pica Ciamarra M, Lara AH, Lee AT, Goldman DI, Vishik I, Swinney HL. 2004 Dynamics of drag and force distributions for projectile impact in a granular medium. Phys. Rev. Lett 92, 194301.

[2] Ricci L, Nguyen VH, Sab K, Duhamel D, Schmitt L. 2005 Dynamic behaviour of ballasted railway tracks: A discrete/continuous approach. Computers and Structures 83, 2282-2292.

[3] Nesterenko VF. 2003 Shock (blast) mitigation by "soft" condensed matter. In Granular Material-Based Technologies, Proc. MRS Symp., 759 (eds Sen S, Hunt ML, Hurd AJ), pp. MM4.3.1-4.3.12. Pittsburgh, PA: MRS Press. (doi:10.1557/PROC-759-MM4.3).

[4] Fraternali F, Porter MA, Daraio C. 2010 Optimal design of composite granular protectors. Mech. Adv. Mat. Struct. 17, $1-19$.

[5] Porter M, Kevrekidis PG, Daraio C. 2015 Granular crystals : Nonlinear dynamics meets materials engineering. Physics Today 68, 44.

[6] Starosvetsky Y, Jayaprakash KR, Hasan MA, Vakakis AF. 2017 Topics on the Nonlinear Dynamics and Acoustics of Ordered Granular Media. Singapore: World Scientific.

[7] Burgoyne HA, Newman JA, Jackson WC, Daraio C. 2015 Guided impact mitigation in 2D and 3D granular crystals. Procedia Engineering 103, 52-59.

[8] Nguyen N-S, Brogliato B. 2014 Multiple Impacts in Dissipative Granular Chains. Lecture Notes in Applied and Computational Mechanics, no. 72. Berlin, Germany: Springer.

[9] Hinch EJ, Saint-Jean S. 1999 The fragmentation of a line of balls by an impact. Proc. R. Soc. Lond. A 455, $3201-3220$.

[10] Nesterenko VF. 2001 Dynamics of Heterogeneous Materials. Berlin, Germany: Springer.

[11] Sen S, Hong J, Bang J, Avalos E, Doney R. 2008 Solitary waves in the granular chain. Physics Reports 462, 21-66.

[12] Jayaprakash KR, Starosvetsky Y, Vakakis AF. 2011 New family of solitary waves in granular dimer chains with no precompression. Phys. Rev. E 86, 036606.

[13] James G, Pelinovsky P. 2014 Gaussian solitary waves and compactons in Fermi-Pasta-Ulam lattices with Hertzian potentials. Proc. R. Soc. A. 470 (2165), 20130462.

[14] Vorotnikov K, Starosvetsky Y, Theocharis G, Kevrekidis PG. 2018 Wave propagation in a strongly nonlinear locally resonant granular crystal. Physica D 365, 27-41.

[15] James G, Kevrekidis PG, Cuevas J. 2013 Breathers in oscillator chains with Hertzian interactions. Physica D 251, 39-59. 
[16] Pfeiffer F, Glocker C. 1996 Multibody Dynamics with Unilateral Contacts, Wiley Series in Nonlinear Science, John Wiley \& Sons, Inc.

[17] Brogliato B. 2016 Nonsmooth Mechanics: Models, Dynamics and Control. Communications and Control Engineering. Third Edition. Springer International Publishing, Switzerland.

[18] Nguyen N-S, Brogliato B. 2018 Comparisons of multiple-impact laws for multibody systems: Moreau's law, binary impacts, and the LZB approach. In Advanced Topics in Nonsmooth Dynamics (eds Leine RI, Acary V, Brüls O), Springer International Publishing AG, Cham, Switzerland, p. 1-45

[19] Johnson KL. 1985 Contact Mechanics, Cambridge Univ. Press, Cambridge.

[20] Acary V, Brogliato B. 2004 Coefficients de restitution et efforts aux impacts : Revue et comparaison des estimations analytiques. [Rapport de recherche] RR-5401, INRIA, pp.162. ¡inria-00070602i

[21] Falcon E, Laroche C, Fauve S, Coste C. 1998 Behavior of one inelastic ball bouncing repeatedly off the ground. Eur. Phys. J. B 3, 45-57.

[22] Lee J, Herrmann HJ. 1993 Angle of repose and angle of marginal stability: molecular dynamics of granular particles. $J$. Phys. A: Math. Gen. 26, 373-383.

[23] Simon R. 1967 The development of a mathematical tool for evaluating golf club performance. Proceedings of ASME Design Engineering Conference, New York city, USA.

[24] Hunt KH, Crossley F.E. 1975 Coefficient of restitution interpreted as damping in vibroimpact. Journal of Applied Mechanics 42, 440-445.

[25] Kuwabara G, Kono K. 1987 Restitution coefficient in a collision between two spheres. Japanese Journal of Applied Physics 26, 1230-1233.

[26] Zener C. 1941 The intrinsic inelasticity of large plates. Physical Review 59, 669-673.

[27] Liu C, Zhao Z, Brogliato B. 2008 Frictionless multiple impacts in multibody systems. I. Theoretical framework. Proc. R. Soc. A 464 (2100), 3193-3211.

[28] Liu C, Zhao Z, Brogliato B. 2009 Frictionless multiple impacts in multibody systems. II. Numerical algorithm and simulation results. Proc. R. Soc. A 465 (2101), 1-23.

[29] Pal RK, Awasthi AP, Geubelle PH. 2013 Wave propagation in elasto-plastic granular systems. Granular Matter 15, 747-758.

[30] Burgoyne HA, Daraio C. 2015 Elastic-plastic wave propagation in uniform and periodic granular chains. Journal of Applied Mechanics 82, 081002.

[31] Tatara Y, Moriwaki N. 1982 Study on impact of equivalent two bodies (coefficients of restitution of spheres of brass, lead, glass, porcelain and agate, and the material properties). Bulletin of JSME 25, No 202, 631-637.

[32] Zhang Y, Sharf I. 2009 Validation of nonlinear viscoelastic contact force models for low speed impact. Journal of Applied Mechanics 76, 051002.

[33] Kačianauskas R, Kruggel-Emden H, Zdancevičius E, Markauskas D. 2016 Comparative evaluation of normal viscoelastic contact force models in low velocity impact situations. Advanced Powder Technology 27, 1367-1379.

[34] Hutzler S, Delaney G, Weaire D, MacLeod F. 2004 Rocking Newton's cradle. Am. J. Phys. 72, 1508-1516.

[35] Carretero-González R, Khatri D, Porter MA, Kevrekidis PG, Daraio C. 2009 Dissipative solitary waves in granular crystals. Phys. Rev. Lett. 102, 024102.

[36] Brilliantov NV, Pimenova AV, Goldobin DS. 2015 A dissipative force between colliding viscoelastic bodies: Rigorous approach. EPL 109, 14005.

[37] Goldobin DS, Susloparov EA, Pimenova AV, Brilliantov NV. 2015 Collision of viscoelastic bodies: Rigorous derivation of dissipative force. Eur. Phys. J. E 38: 55.

[38] Villatoro FR, Ramos JI. 1999 On the method of modified equations. I: Asymptotic analysis of the Euler forward difference method. Applied Mathematics and Computation 103, 111-139.

[39] Hairer E, Lubich C, Wanner G. 2006 Geometric Numerical Integration: Structure-Preserving Algorithms for Ordinary Differential Equations, Springer-Verlag Berlin Heidelberg.

[40] Ascher UM, Petzold LR. 1998 Computer Methods for Ordinary Differential Equations and Differential-Algebraic Equations, SIAM, Philadelphia.

[41] Kennedy CA, Carpenter MH. 2003 Additive Runge-Kutta schemes for convection-diffusion-reaction equations. Applied Numerical Mathematics 44, 139-181.

[42] Acary V. 2016 Energy conservation and dissipation properties of time-integration methods for nonsmooth elastodynamics with contact. Z. Angew. Math. Mech. 96, 585-603.

[43] Ramírez R, Pöschel T, Brilliantov NV, Schwager T. 1999 Coefficient of restitution of colliding viscoelastic spheres. Phys. Rev. E 60, 4465-4472.

[44] Hairer E, Nørsett SP, Wanner G. 1993 Solving Ordinary Differential Equations I: Nonstiff Problems, Springer-Verlag Berlin Heidelberg.

[45] Hairer E, Wanner G. 1991 Solving Ordinary Differential Equations II: Stiff and Differential-Algebraic Problems, Springer-Verlag Berlin Heidelberg. 
[46] Janežič D, Orel B. 1993 Implicit Runge-Kutta method for molecular dynamics integration. J. Chem. Inf. Comput. Sci. 33, 252-257.

[47] Demailly J-P. 2006 Analyse Numérique et Équations Différentielles, Collection Grenoble Sciences, EDP Sciences, Les Ulis.

[48] Hairer E, Lubich Ch. 2000 Asymptotic expansions and backward analysis for numerical integrators. In Dynamics of algorithms (eds de la Llave R, Petzold LR, Lorenz J.), Minneapolis, MN, 1997, IMA Vol. Math. Appl. 118, Springer, New York, 91-106.

[49] Sanz-Serna JM, Calvo MP. 1994 Numerical Hamiltonian Problems, Chapman \& Hall, London.

[50] Hairer E, Stoffer D. 1997 Reversible long-term integration with variable stepsizes. SIAM J. Sci. Comput. 18, 257-269.

${ }^{1}$ InRIA Grenoble - Rhône-Alpes, Tripop Team, Inovallée, 655 AVEnue de l'Europe, 38334 Saint Ismier Cedex, France, ${ }^{2}$ Univ. Grenoble Alpes, CNRS, Inria, Grenoble INP*, LJK, 38000 Grenoble, France. * Institute of ENGINEERING Univ. GRENOble AlPES

E-mail address: guillaume.james@inria.fr, kirill.vorotnikov@gmail.com, bernard.brogliato@inria.fr 